\title{
Long non-coding RNA NEAT1 promotes bone metastasis of prostate cancer through N6-methyladenosine
}

\author{
Simeng Wen ${ }^{1 \dagger}$, Yulei $\mathrm{Wei}^{2 \dagger}$, Chong Zen ${ }^{3}$, Wei Xiong ${ }^{3}$, Yuanjie Niu ${ }^{1 *}$ and Yu Zhao ${ }^{4^{*}}$ (D)
}

\begin{abstract}
Background: N6-methyladenosine (m6A) is the most prevalent messenger RNA modification in mammalian cells. However, the disease relevant function of m6A on specific oncogenic long non-coding RNAs (ncRNAs) is not well understood.
\end{abstract}

Methods: We analyzed the m6A status using patients samples and bone metastatic PDXs. Through m6A highthroughput sequencing, we identified the m6A sites on NEAT1-1 in prostate bone metastatic PDXs. Mass spec assay showed interaction among NEAT1-1, CYCLINL1 and CDK19. RNA EMSA, RNA pull-down, mutagenesis, CLIP, western blot, ChIP and ChIRP assays were used to investigate the molecular mechanisms underlying the functions of m6A on NEAT1-1. LOSS-of function and rescued experiments were executed to detect the biological roles of m6A on NEAT1-1 in the PDX cell phenotypes in vivo.

Results: In this study, we identified 4 credible m6A sites on long ncRNA NEAT1-1. High m6A level of NEAT1-1 was related to bone metastasis of prostate cancer and m6A level of NEAT1-1 was a powerful predictor of eventual death. Transcribed NEAT1-1 served as a bridge to facility the binding between CYCLINL1 and CDK19 and promoted the Pol II ser2 phosphorylation. Importantly, depletion of NEAT1-1or decreased m6A of NEAT1-1 impaired Pol II Ser$2 \mathrm{p}$ level in the promoter of RUNX2. Overexpression of NEAT1-1 induced cancer cell metastasis to lung and bone; xenograft growth and shortened the survival of mice, but NEAT1-1 with m6A site mutation failed to do these.

Conclusion: Collectively, the findings indicate that m6A on ncRNA NEAT1-1 takes critical role in regulating Pol II ser2 phosphorylation and may be novel specific target for bone metastasis cancer therapy and diagnosis. New complex CYCLINL1/CDK19/NEAT1-1 might provide new insight into the potential mechanism of the pathogenesis and development of bone metastatic prostate cancer.

Keywords: Bone metastatic prostate cancer, m6A, NEAT1-1, CYCLINL1, CDK19, nCRNA

\footnotetext{
* Correspondence: niuyuanjie@tmu.edu.cn; zhao.yu@mayo.edu

†Simeng Wen and Yulei Wei contributed equally to this work.

'Department of Urology, The Second Hospital of Tianjin Medical University, Tianjin Medical University, Tianjin 300211, China

${ }^{4}$ Department of Biochemistry and Molecular Biology, Mayo Clinic, Rochester, MN 55905, USA

Full list of author information is available at the end of the article
}

(c) The Author(s). 2020 Open Access This article is licensed under a Creative Commons Attribution 4.0 International License, which permits use, sharing, adaptation, distribution and reproduction in any medium or format, as long as you give appropriate credit to the original author(s) and the source, provide a link to the Creative Commons licence, and indicate if changes were made. The images or other third party material in this article are included in the article's Creative Commons licence, unless indicated otherwise in a credit line to the material. If material is not included in the article's Creative Commons licence and your intended use is not permitted by statutory regulation or exceeds the permitted use, you will need to obtain permission directly from the copyright holder. To view a copy of this licence, visit http://creativecommons.org/licenses/by/4.0/ The Creative Commons Public Domain Dedication waiver (http://creativecommons.org/publicdomain/zero/1.0/) applies to the data made available in this article, unless otherwise stated in a credit line to the data. 


\section{Introduction}

Prostate cancer is the most common malignancy among males in the world, and also is the second-most common cause of cancer death in United States (US) males $[1,2]$. Metastasis is main cause of death in prostate cancer. Prostate cancer has an affinity to metastasize to bone, about $80 \%$ of the metastatic prostate cancer cells spread to bones, such as the hip, spine, and pelvis bones $[3,4]$. With androgen sensitive advanced prostate cancer, approximately $50 \%$ of men will develop bone metastases within two years [5]. 56\% of prostate cancer patients without bone metastases were alive at five years, compared to $3 \%$ of patients with bone metastases. It suggests that bone metastatic prostate cancer is a poor prognosis in men and correlates with significant mortality [6]. Prostate cancer invades or travels through your blood or lymphatic system and once relocated, the cells begin to grow as "new" tumors. The molecular event for bone metastatic tumor remains unclear.

Over 100 types of cellular RNA modifications, N6methyladenosine (m6A) is the most abundant posttranscriptional RNA modification on eukaryote RNAs [7-9]. The functions of m6A in mRNA include nuclear transport, splicing, stability and translation [10-14]. However, the m6A function in long non-coding RNA is limit to report. The nuclear-enriched abundant transcript 1(NEAT1) is an overexpressed long non-coding RNA in many human cancer types [15]. The higher expression of NEAT1 is significantly associated with worse survival in cancer patients $[15,16]$. NEAT1 is responsible for several cancer clinical features: patient survival, metastasis, recurrence, stem cell-like phenotype [16]. Knockdown of NEAT1 can decrease the malignant behavior of tumor cells for breast cancer [17, 18], hepatocellular carcinoma [19], laryngeal squamous cell carcinoma [20], lung cancer [21], glioma [22], prostate cancer [23], and skin cancer [24]. NEAT1 is identified as an essential component of nuclear paraspeckles [25] and mediates transcription [26, 27]. NEAT1 is transcribed from familial tumor syndrome multiple endocrine neoplasia type I loci and encodes two transcriptional variants, NEAT1-1 ( $3.7 \mathrm{~kb})$ and NEAT1-2 ( 22.7 kb) [28]. NEAT1-1 serves as the ER $\alpha$-inducible oncogenic target and promotes the cancer progression in castrateresistant prostate cancer (CRPC) [23]. On the contrary, NEAT1-1 can be regulated by $\mathrm{p} 53$ to suppress the cancer transformation in pancreatic cancer $[29,30]$.

In this study, we identified the novel m6A function in ncRNA that played an oncogenic role in bone metastatic prostate cancer and was correlated with poor prognosis. Further experiments demonstrated that NEAT1-1 m6A facilitated the oncogenic function of new complex CYCL INL1/CDK19 for Pol II Ser2 phosphorylation. The regulatory network involving the new complex CYCLINL1/
CDK19/NEAT1-1 might provide new insight into the potential mechanism of the pathogenesis and development of bone metastatic prostate cancer.

\section{Methods}

Plasmids, reagents and antibodies

GST-tagged Pol II-C terminal were generated by cloning the corresponding cDNAs into pGEX-4 T-1 vector. The cDNA fragments were amplified by Phusion polymerase (NEB, USA) using Phusion High-Fidelity PCR Master Mix. pCRII-NEAT1-1 was kind gift from Dr. Xiong in Second Xiangya Hospital [31]. Mammalian expression plasmids for CYCLINL1, CDK19 and NEAT1-1 were sub-generated by inserting each target cDNA into the pcDNA3.1 vector. The insert and deletion mutants were constructed using QuikChange II Site directedMutagenesis Kit (Agilent, USA). Antibodies and primers were shown in Table S1.

\section{Cell lines, cell culture and reagents}

Short term explant cultures were obtained from a primary patient derived bone metastatic prostate panel. PDXs related primary cells were cultured in RPMI 1640 medium supplemented with $10 \%$ FBS or charcoalstripped fetal bovine serum (FBS) (Invitrogen) (androgen-depleted medium) and $100 \mu \mathrm{g} / \mathrm{ml}$ penicillinstreptomycin-glutamine (Invitrogen) at $37^{\circ} \mathrm{C}$ with $5 \%$ $\mathrm{CO}_{2}$. Cell authentication was performed using STR profiling as previously description [32, 33].

\section{Human prostate cancer specimens and RNA isolation from human tissues}

Formalin-fixed paraffin-embedded (FFPE) or freshfrozen hormone-naïve primary prostate cancer and bone metastatic tissues were randomly selected from the Tianjin Medical University Tissue Registry and Second Xiangya Hospital. Hormone-naïve patients with biopsyproven prostate cancer have been treated at Tianjin Medical University by radical retropubic prostatectomy between January 1995 and December 2018 without neoadjuvant therapy. The clinical tissues were approved by the Tianjin Medical University Institutional Review Board and Medical Ethics Committee. FFPE tissues were collected and total RNAs were isolated using a RecoverAll Total Nucleic Acid Isolation Kit (Life Technologies). Isolation of RNAs from frozen human prostate cancer tissues was performed as described previously [34].

\section{Patient-derived xenograft (PDX)}

PDXs were established through injection of surgical tissues into the flank of male athymic nude mice 6-8 weeks of age. The procedures for establishment and maintenance of flank xenografts and short-term explant cultures were followed the published procedures 
from Mayo Clinic Patient-Derived Xenograft National Resource Center [35]. P-18 is the Number 18 of 66 PDXs in Tianjin Medical University, and is the orthotopic metastasis prostate cancer tissue from a 76 years old patient's right pelvis after a surgery. P-34 is the Number 34 of 66 PDXs in Tianjin Medical University, and is the orthotopic metastasis prostate cancer tissue from an 86 years old patient after a surgery. Mice were housed in the Tianjin Medical University pathogen-free rodent facility. All procedures were approved by the Tianjin Medical University Institutional Animal Care and Use Committee.

\section{Mouse xenograft generation and tumor growth measurement}

The mouse study was approved by Tianjin Medical University Institutional Animal Care and Use Committee. Six-week- old NSG male mice were injected with $5 \times 10^{6}$ of cancer cells infected with lentivirus or shRNAs and/or expression vectors in $100 \mu \mathrm{l}$ PBS with $100 \mu \mathrm{l}$ of Matrigel matrix (BD Bioscience) in right flanks. After injection of tumor cells into mice, tumors were monitored until they reach maximum tumor volumes of $1000 \mathrm{~mm}^{3}$ and tumor growth was measured with caliper every 6 days.

\section{Mouse tail-vein injection of tumor cells and \\ bioluminescent imaging}

The mouse study was approved by Tianjin Medical University Institutional Animal Care and Use Committee. Male NSG mice were used for the experiment. P-18 cells stably expressing the luciferase genes were infected with lentivirus for empty vector or NEAT1-1 WT and m6A-\#4 site mutants. $1 \times 10^{6}$ infected cells in $0.2 \mathrm{ml}$ PBS were injected via tail vein into individual mice (six mice each group). Mice were monitored by bioluminescent imaging. Mice were injected with luciferin $(300 \mathrm{mg} / \mathrm{kg}$ ) $10 \mathrm{~min}$ before imaging. Mice were anaesthetized (3\% isoflurane) and imaged using the IVIS spectrum imaging system (Xenogen, Life Sciences). Images were analyzed with Living Image software (Xenogen, Life Sciences). Bioluminescent flux (photons $/ \mathrm{s} / \mathrm{sr} / \mathrm{cm}^{2}$ ) was determined for lesions in lung and body.

\section{In vitro transcription and RNA pulldown by GST recombinant proteins}

A fragment or full length, corresponding to the NEAT11 RNA, was subcloned into the pcDNA3.1 backbone vector. Mutations with various deletions within this region were generated by mutagenesis using a KODPlus-Mutagenesis Kit (TOYOBO). Myc-CYCLINL1 and Flag-CDK19 were translation using TNT Quick coupled transcription/translation system kit (Promege). Wildtype and mutated vectors were linearized by digestion with XhoI and purified by the Gel Extraction Kit
(Qiagen). $200 \mathrm{ng}$ linearized plasmid DNAs were transcribed in vitro using T7 RNA polymerase. RNAs were treated with DNase I to eliminate the template DNA. GST-Pol II-C terminal recombinant proteins were expressed in E. coli (BL21) after induction with $0.5 \mathrm{mM}$ IPTG at $16^{\circ} \mathrm{C}$ for $12 \mathrm{~h}$ and purified with glutathione Sepharose 4B beads (GE Healthcare) as described previously [34]. Purified GST recombinant proteins were incubated with in vitro transcribed RNA in RNA structure buffer $(50 \mathrm{mM}$ Tris, pH 7.4, $150 \mathrm{mM} \mathrm{NaCl} ; 1 \mathrm{mM}$ $\mathrm{MgCl}_{2}$ ) [34]. For the GST-Pol II-C binding assay, ATP and $\mathrm{MgCl}_{2}$ was added to the binding buffer to a final concentration of $1 \mathrm{mM}$. After extensive washes, RNAs were purified using the RNeasy MinElute Cleanup Kit (Qiagen). Precipitated RNA was detected by real-time RT-PCR.

\section{Design and screening of highly optimized generation-2.5 antisense oligonucleotides (ASOs)}

The ASOs used in this study contained a full phosphorothioate backbone and a 10-base 2'-deoxynucleoside gap flanked by 2 '-O-methyl (cMt)-modified nucleotides. The motif for the ASOs targeting the NEAT1-1 RNA tested was $\mathrm{mmm}-10-\mathrm{mmm}$, where $\mathrm{m}$ represents cMt modification and - 10- represents the 10-base DNA gap. ASOs were synthesized and purified as described previously [36]. A large number of ASOs targeting sense-strand NEAT1-1 RNA were screened by Rebio Pharmaceuticals Inc. (Guangzhou, China) for high efficient reduction of RNA. ASO1 (5'- GAGTGATGTGGAGTTA-3') and ASO2 (5' - GGCTCTTCTGGATTTG-3') that resulted in strong reduction of NEAT1-1 RNA were selected for further studies. Negative control ASO (5'-GGCTACTACG CCGTCA-3') with the same chemistry, but matching no human transcripts, Control were included in each experiment to demonstrate the specificity of NEAT1-1 ASOs. These oligonucleotides were designed to exclude Gstrings with four Gs or two sets of three Gs in a row to prevent non-antisense-mediated effects [34].

\section{RNA isolation from cultured cells, reverse transcription PCR (RT-PCR) and real-time PCR}

Total RNA was isolated from cultured cells using TRIzol reagent (Invitrogen) or the RNeasy Plus Mini Kit (Qiagen) according to the manufacturer's instructions. First-strand cDNA was synthesized with the PrimeScript Reverse Transcriptase Kit (TaKaRa Bio). Reverse transcription and real-time PCR were performed as described previously [37]. The PCR primers for AR new target genes are listed in Table S1.

Biotin-labeled RNA pulldown and western blot analysis RNAs were biotin-labeled during in vitro transcription using Biotin RNA Labeling Mix (Roche) and T7 
polymerase (New England Biolabs). P-18 primary cells cultured in androgen-depleted medium were lysed in modified Binding buffer (50 mM Tris- $\mathrm{HCl}$ pH 7.5, 150 $\mathrm{mM} \mathrm{NaCl}, 1 \% \mathrm{NP}-40,0.1 \%$ SDS and $1 \%$ protease inhibitor cocktails). Cell lysates were incubated with biotinlabelled RNAs and streptavidin beads at $4{ }^{\circ} \mathrm{C}$ for $12 \mathrm{~h}$. The beads were washed in wash buffer $(50 \mathrm{mM}$ Tris, $\mathrm{pH}$ 7.4; $150 \mathrm{mM} \mathrm{NaCl}$; 0.05\% Nonidet P-40 (NP-40); $1 \mathrm{mM}$ $\mathrm{MgCl} 2)$ at $4{ }^{\circ} \mathrm{C}$ six times. The samples were subjected to western blot analyses as described previously [34]. Briefly, protein samples were denatured and subjected to SDS-polyacrylamide gel electrophoresis (SDS/PAGE), and were transferred to nitrocellulose membranes (BioRad). The membranes were immunoblotted with specific primary antibodies, horseradish peroxidase-conjugated secondary antibodies, and visualized by SuperSignal West Pico Stable Peroxide Solution (Thermo Scientific). The antibodies are shown in Table S1.

\section{Chromatin immunoprecipitation (ChIP)}

ChIP was performed as described previously [34]. The online Biosearch Technologies' Stellaris FISH Probe Designer was used to design antisense oligo probes tiling NEAT1-1 RNA. The probe oligos were synthesized with a 3 '-Biotin-TEG modification and purified by HPLC.

\section{m6A RIP-seq and data analysis}

The m6A RIP-seq data were analyzed from GSE63753 [38]. Pre-analysis quality control was performed using FastQC (http://www.bioinformatics.babraham.ac.uk/projects/fastqc/) and RSeQC software [39] to ensure that raw data are in excellent condition and suitable for downstream analyses. Pair-end raw reads were aligned to the human reference genome (GRch37/hg19) using Tophat [40]. Genome-wide coverage signals were represented in BigWig format to facilitate convenient visualization using the UCSC genome browser. Gene expression was measured using RPKM (Reads Per Kilo-base exon per Million mapped reads) as described previously [40]. Correlation analyses between RNA expressions were performed using Python and R scripts.

\section{RNA-seq and data analysis}

P18 cells or P18-NEAT1-1 KO cells were transfected with ASO or NEAT1-1 plasmids for $48 \mathrm{~h}$. Total RNAs were isolated from cells using the methods as described previously [34]. Briefly, RNA was isolated using RNeasy Plus Mini Kit (Qiagen). High quality total RNAs (Agilent Bioanalyzer RIN > 7.0) were employed for the preparation of sequencing libraries using Illumina TruSeq Stranded Total RNA/Ribo-Zero Sample Prep Kit. A total of 500-1000 ng of riboRNA-depleted total RNA was fragmented by RNase III treatment at $37^{\circ} \mathrm{C}$ for $20 \mathrm{~min}$ and RNase III was inactivated at $65^{\circ} \mathrm{C}$ for $10 \mathrm{~min}$. Size selection (50 to $150 \mathrm{bp}$ fragments) was performed using the FlashPAGE denaturing PAGE-fractionator (Life Technologies) prior to ethanol precipitation overnight. The resulting RNA was directionally ligated, reversetranscribed and RNase $\mathrm{H}$ treated. Samples with biological duplicates were sequenced using the Illumina HiSeq2000 platform at the BGI Genomics (Shenzhen China). Pre-analysis quality control was performed using FastQC and RSeQC software to ensure that raw data are in excellent condition and suitable for downstream analyses. Pair-end raw reads were aligned to the human reference genome (GRch37/hg38) using Tophat. Genomewide coverage signals were represented in BigWig format to facilitate convenient visualization using the UCSC genome browser. Gene expression was measured using RPKM (Reads Per Kilo-base exon per Million mapped reads). Correlation analyses between mRNA expressions in different groups were performed using Python and $\mathrm{R}$ scripts. EdgeR was used to identify genes that were differentially expressed between NEAT1-1 knocking down or m6A-deletion.

\section{Chromatin immunoprecipitation (ChIP) and chromatin isolation by RNA purification (ChIRP)}

ChIP was performed as described previously [34]. The ChIRP experiment was performed essentially as per the original protocol as described previously [34]. The online Biosearch Technologies' Stellaris FISH Probe Designer was used to design antisense oligo probes tiling NEAT11 RNA. The probe oligoes were synthesized with a 3 'Biotin-TEG modification and purified by HPLC. Two pools of probes were prepared, one with $10 \mu \mathrm{M}$ of each even numbered probe $(2,4,6$, etc.) and the other with $10 \mu \mathrm{M}$ of each odd numbered probe (1, 3, 5, etc.).The ChIP primers and ChIRP probes are shown in Table S1.

\section{Clustered regularly interspaced short palindromic repeats (CRISPR)-Cas9 system}

CRISPR-Cas9 assay was performed using CRISPR-Cas9 tool kit (Santa Cruz). gRNAs were cloned into lentivirusV2 plasmid, under U6 rpomoter. The gRNAs are shown in Table S1.

RNA electrophoretic mobility shift assay (RNA EMSA)

Biotin-labeled RNA probes were generated by in vitro transcription using cDNA containing $\mathrm{T} 7$ promoter and the NEAT1-1 fragment RNA were purchased from BGI Genomics (Shenzhen, China). For the RNA EMSA assay, recombinant Myc-CYCLINL1 or Flag-CDK19, $100 \mathrm{ng} / \mathrm{ml}$ tRNA, and $1 \mathrm{l} \mu \mathrm{g}$ of biotin-labeled RNA probe were mixed in binding buffer $(10 \mathrm{mM}$ Tris-Cl, $\mathrm{pH} 7.5,25 \mathrm{mM} \mathrm{KCl}$, $10 \mathrm{mM} \mathrm{MgCl} 2,1 \mathrm{mM} \mathrm{DTT}$ ) for $30 \mathrm{~min}$ at $25^{\circ} \mathrm{C}$. RNAprotein complexes were digested by adding 100 units of RNase-T1 for $15 \mathrm{~min}$ at $37^{\circ} \mathrm{C}$ and then sepa- rated in $6 \%$ 
of native poly acrylamide gel. RNA-protein complexes were blotted with HRP-conjugated streptavidin and the final results were visualized by autoradiography.

Co-immunoprecipitation (co-IP) and Western blot analysis To immunoprecipitate the ectopically expressed Flag or Myc-tagged proteins, transfected cells were lysed $24 \mathrm{~h}$ post-transfection in BC100 buffer. The whole-cell lysates were immunoprecipitated with the monoclonal anti-Flag or anti-Myc antibody-conjugated agarose beads (SigmaAldrich) at $4{ }^{\circ} \mathrm{C}$ overnight. After three washes with lysis buffer, followed by two washes with BC100 buffer, the bound proteins were eluted using Myc or Flag-Peptide (Sigma-Aldrich) prepared in $\mathrm{BC} 100$ for $3 \mathrm{~h}$ at $4{ }^{\circ} \mathrm{C}$. The eluted protein sample was resolved by SDS-PAGE. Cells cultured in androgen-depleted medium were lysed in modified Binding buffer (50 mM Tris- $\mathrm{HCl}$ pH 7.5, 150 $\mathrm{mM} \mathrm{NaCl}, 1 \% \mathrm{NP}-40,0.1 \% \mathrm{SDS}$ and $1 \%$ protease inhibitor cocktails). Cell lysates were incubated with biotinlabelled RNAs and streptavidin beads at $4{ }^{\circ} \mathrm{C}$ for $12 \mathrm{~h}$. The beads were washed in wash buffer $(50 \mathrm{mM}$ Tris, $\mathrm{pH}$ 7.4; $150 \mathrm{mM} \mathrm{NaCl}$; 0.05\% Nonidet P-40 (NP-40); $1 \mathrm{mM}$ $\mathrm{MgCl} 2$ ) at $4{ }^{\circ} \mathrm{C}$ six times. Briefly, protein samples were denatured and subjected to SDS-polyacrylamide gel electrophoresis (SDS/PAGE), and were transferred to nitrocellulose membranes (Bio-Rad). The membranes were immunoblotted with specific primary antibodies, horseradish peroxidase-conjugated secondary antibodies, and visualized by SuperSignal West Pico Stable Peroxide Solution (Fisher). The antibodies are shown in Table S1.

\section{Pol II CTD kinase assay}

GST and GST-Pol II-C terminal domain (GST-Pol II-C, 372 amino acids of the Pol II-C terminal domain) recombinant proteins were incubated with CDK19 and CYCLINL1 translation products for kinase assay using the method as described previously [34]. The kinase reaction mixtures were incubated at $30{ }^{\circ} \mathrm{C}$ for $60 \mathrm{~min}$ and then stopped by the addition of SDS-PAGE buffer. The samples were measured using western blotting.

\section{RNA dot blot hybridization}

The dot blot assays were performed as described previously [34, 37]. Briefly, equal amounts of biotin-labelled RNA were dot blotted and the blot was incubated with the Streptavidin-HRP antibody for $2 \mathrm{~h}$ and visualized by SuperSignal West Pico Stable Peroxide Solution (Thermo Scientific).

\section{RNA fluorescence in situ (FISH)}

RNA FISH and imaging were performed as described previousely [41]. The probes targeting NEAT1-1, plasmids, and AR were designed by probe design software online (https:/www.biosearchtech.com/stellaris-designer) and synthesized by BGI Genomics (Shenzhen, China). The probes were list in Table S1.

\section{Cross-linking immunoprecipitation (CLIP) and individual- nucleotide resolution CLIP (iCLIP)}

$5 \times 10^{6}$ cells treated with $100 \mu \mathrm{M}$ 4-Thiouridine (4SU) for $8 \mathrm{~h}$ were washed with cold PBS one time and cells were irradiated once with $150 \mathrm{~mJ} / \mathrm{cm} 2$ at $365 \mathrm{~nm}$ using a Stratalinker. Cells were lysed in lysis buffer $(50 \mathrm{mM}$ Tris-HCL, pH 7.4, 100 mM NaCl, 1\% NP-40, 0.1\% SDS, $0.5 \%$ sodium deoxycholate, protease inhibitor cooktail and RNase inhibitors) with protease inhibitors $(1 \mathrm{~mL})$ and transferred to $1.5 \mathrm{~mL}$ microtubes. Lysate was partially digested by $2 \mathrm{U} / \mu \mathrm{L}$ RNaseT1/A for $15 \mathrm{~min}$ at $22^{\circ} \mathrm{C}$ for iCLIP. RNA was immunoprecipitated with CYCL INL1 or CDK19 antibodies and protein A/G beads for 4 $\mathrm{h}$ at $4{ }^{\circ} \mathrm{C}$. After washed for 4 times, RNA was phosphorylated by T4 PNK and ligated RNA between $3^{\prime}$ and 5' ends by RNA T4 ligase. SDS-PAGE loading buffer was added and the mixture was incubated at $70{ }^{\circ} \mathrm{C}$ for 10 min. After running the SDS-PAGE gel, the RNA-protein complexes were transferred from gel to a nitrocellulose membrane using a wet transfer apparatus ( $30 \mathrm{~V}$ for $1 \mathrm{~h}$ ). The membrane with target protein was cut up, and the targeted membrane piece was incubated with Proteinase $\mathrm{K}$ for de-crosslink. After de-crosslink, RNA was reverse transcribed into cDNA and subjected to real-time qPCR analysis.

\section{Statistical analysis}

For in vivo experiments, animals were randomized. Randomization was not performed for all other experiments. All of the experiments were performed in biological triplicate unless otherwise specified. Statistical analyses were performed using $\mathrm{R}$ Script. Statistical analyses were performed using Student's t test, or two-way ANOVA tests as indicated. $P<0.05$ is considered statistically significant. Non-parametric Kolmogorov-Smirnov (KS) test was used to evaluate statistical significance of differential expression between primary prostate cancer and bone metastatic prostate cancer. Details regarding the statistical methods employed during RNA-seq and m6A RIP-seq data analyses were mentioned in aforementioned methods.

\section{Results}

m6A of NEAT1-1 is elevated in prostate cancer and is a negative prognostic factor for patients

NEAT1-1 as an oncogenic ncRNA is highly expression in multiple cancers. To understand the role of NEAT1-1 in prostate cancer, we queried The Cancer Genome Atlas (TCGA; http://www.cbioportal .org) datasets. In the dataset, NEAT1-1 expression was significantly higher in prostate cancer compare to normal tissues 
(Fig. S1a). Furthermore, elevated expression of NEAT11 predicts poor patient prognosis (Fig. S1b). These data were consistent with pervious reported [23]. To further study the m6A modification of NEAT1-1 in a clinicopathologically relevant context, we harvest the RNA immunoprecipitated by m6A antibody from fresh sample of adjacent normal cancer, primary cancer, lymph nodemetastasis cancer and bone-metastatic cancer. Box plot analysis showed that m6A level of NEAT1-1 was significantly higher in bone metastatic prostate cancer samples than primary prostate cancer or adjacent normal samples in two independent data sets with FFPE samples (Fig. 1a and b) and data set with frozen samples (Fig. S1c). Table 1 showed that m6A levels of NEAT1-1 were statistically significant positive correlations with tumor metastasis and prostate specific antigen (PSA) recruitment, but not with the age, tumor stage and Gleason score. To further substantiate the survival significance of m6A on NEAT1-1, we analyzed the NEAT1-1 expression, m6A level of NEAT1-1 and its correlations with clinical behaviors of prostate cancer patients in our bone-metastasis cohort. Kaplan-Meier survival analysis of Tianjin Medical University data sets showed that high levels of NEAT1-1 or m6A on NEAT1-1 were associated with a shorter survival in prostate cancer patients with bone metastasis and overall survival in primary prostate cancer patients (Fig. 1c and d). Collectively, there was a correlation between NEAT1-1 expression/ m6A status and overall survival.

\section{Four m6A sites were identified in NEAT1-1 in prostate cancer}

The m6A site is usually on the $3^{\prime}$ untranslated region (UTR) of the mRNA like NEAT1's neighbor gene FRMD8 (Fig. S2a). To determine the real m6A site on the NEAT1-1, we search the m6A site via m6A RIP-seq data in cell. From the m6A RIP-seq data in $293 \mathrm{~T}$ cells by two independent m6A antibodies [38, 42], we found that there were 4 high peak on the NEAT1-1 variant 1 (NEAT1-1), and low signals in other regions in NEAT1 variant 2 (NEAT1-2) (Fig. 2a). In addition, we also found that RNA level of NEAT1-1 was approximated 5 fold higher than NEAT1-2 in P-18 cells (Fig. S2b). The m6A RIP data showed that m6A of NEAT1-1 was approximate 10 fold higher than NEAT1-2 in P-18 cells (Fig. S2b). Thus, we focused on the m6A of NEAT1-1. From $5^{\prime}$ to $3^{\prime}$ of NEAT1-1, we labeled the m6A sites from \#1 to \#4 (Figure S2c-f). Based on the conservative motif of m6A site, we found these sites inside the peaks and confirmed these sites by m6A RIP. The crosslinking immunoprecipitation (CLIP) polymerase chain reaction (PCR) assay showed that all four sites had the significantly higher m6A signals than the neighbor sites in prostate cancer P-18 cell (Fig. 2b).
Unlike the messenger RNA (mRNA) FRMD8 (Fig. $\mathrm{S} 2 \mathrm{a}$ ), we found that the $4 \mathrm{~m} 6 \mathrm{~A}$ sites were distributed in whole region from $5^{\prime}$ to $3^{\prime}$ of $N E A T 1-1$. It suggest that m6A on ncRNA may have a different function from mRNA. Next, we further want to know these m6A sites can affect the secondary structure of NEAT1-1, we used the RNA secondary structure prediction software ViennaRNA (https://rna.tbi.univie.ac.at) to predict the secondary structure of NEAT1-1. The prediction figure (Fig. S2c-f) showed that m6A sites \#1, \#2, \#3 were inside the single strand, suggesting m6A modification can't affect the double strand RNA interaction and secondary structure. The $\mathrm{m} 6 \mathrm{~A}$ site \#4 in the double strand and m6A modification may block the double strand RNA interaction here (Fig. S2e). It was noticed that the site\#4 region of NEAT1-1 is a structural flexible region in published data $[43,44]$, suggesting that this region may take different secondary structure for different biological function. To know whether m6A mutation affects the half-life of NEAT1-1 RNA, we measured the half-life of NEAT1-1 WT and site\#4-mut in P-18 cell. The data showed that NEAT1-1 WT half-life time was about 18 min in $293 \mathrm{~T}$ cell, consistent with other reports [45-47]. The half-life of NEAT1-1 WT and site\#4-mut were approximated $8 \mathrm{~h}$ and they were similar with each other (Fig. S2g). The data further showed that the half-life times of NEAT1-1 in P-18 parental and METTL3 knocking-out $(\mathrm{KO})$ cells had no significant difference (Fig. S2g). These data indicated that there were 4 definite m6A sites on NEAT1-1 and site\#4 may affect the secondary structure of NEAT1-1.

\section{NEAT1-1 interacted with CYCLINL1 through m6A site \#4}

To find the molecular function for m6A on NEAT1-1, we generated NEAT1-1 wild type (WT) and m6A deletion mutation plasmid for unbiased tandem affinity purification by biotin labeled probe and mass spectrometry (mass spec). Mass spec assay showed that NEAT1-1 WT bound multiple proteins, including CYCLINL1, but not m6A mutation in primary bone metastatic P-18 cell (Fig. 2c and Table S2). Interestingly, we found multiple pathways related metastasis using Gene Ontology (GO) and Kyoto Encyclopedia of Genes and Genomes (KEGG) analyses, including the pathways were related to extracellular exosome, myelin sheath and lamin filament (Fig. 2d). To confirm the mass spec data, we used biotin labeled probe to target NEAT1-1 to pull down interacted protein. The western blot data showed that only CYCL INL1 bound to NEAT1-1, but not CYCLINT1 and CYCLINC (Fig. 2e). To determine the m6A's role in interaction with CYCLINL1, we knocked down the m6A writer METTL3 in P-18 cells (Fig. S3a). We observed that CYCLINL1 associated with NEAT1-1 in P-18 cells by CLIP assay, but did not associate with NEAT1-1 in 


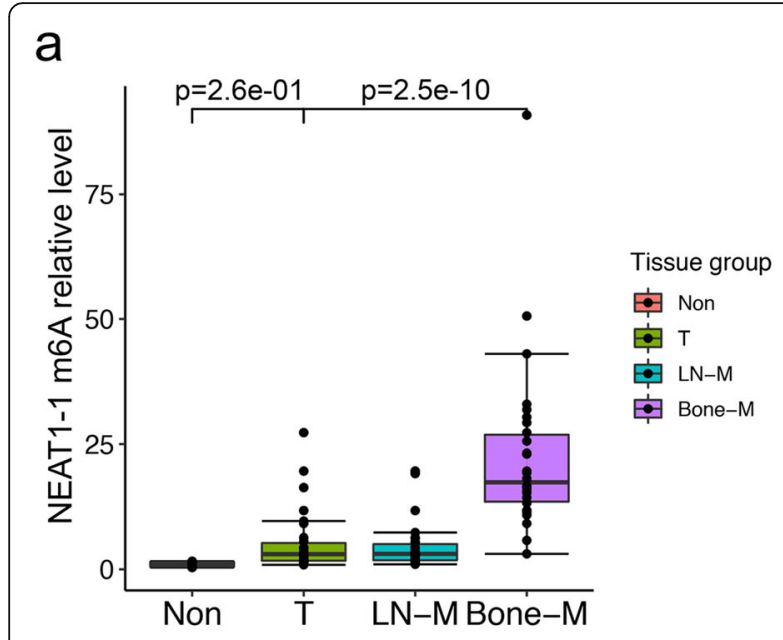

b

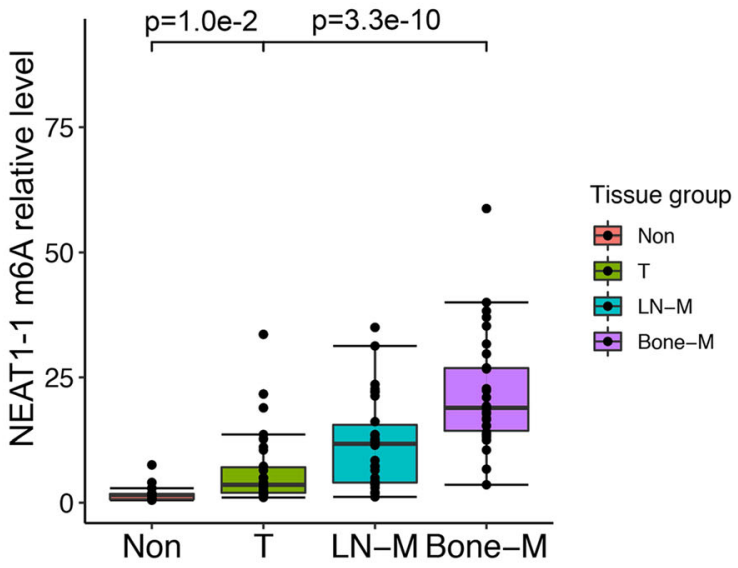

C

d
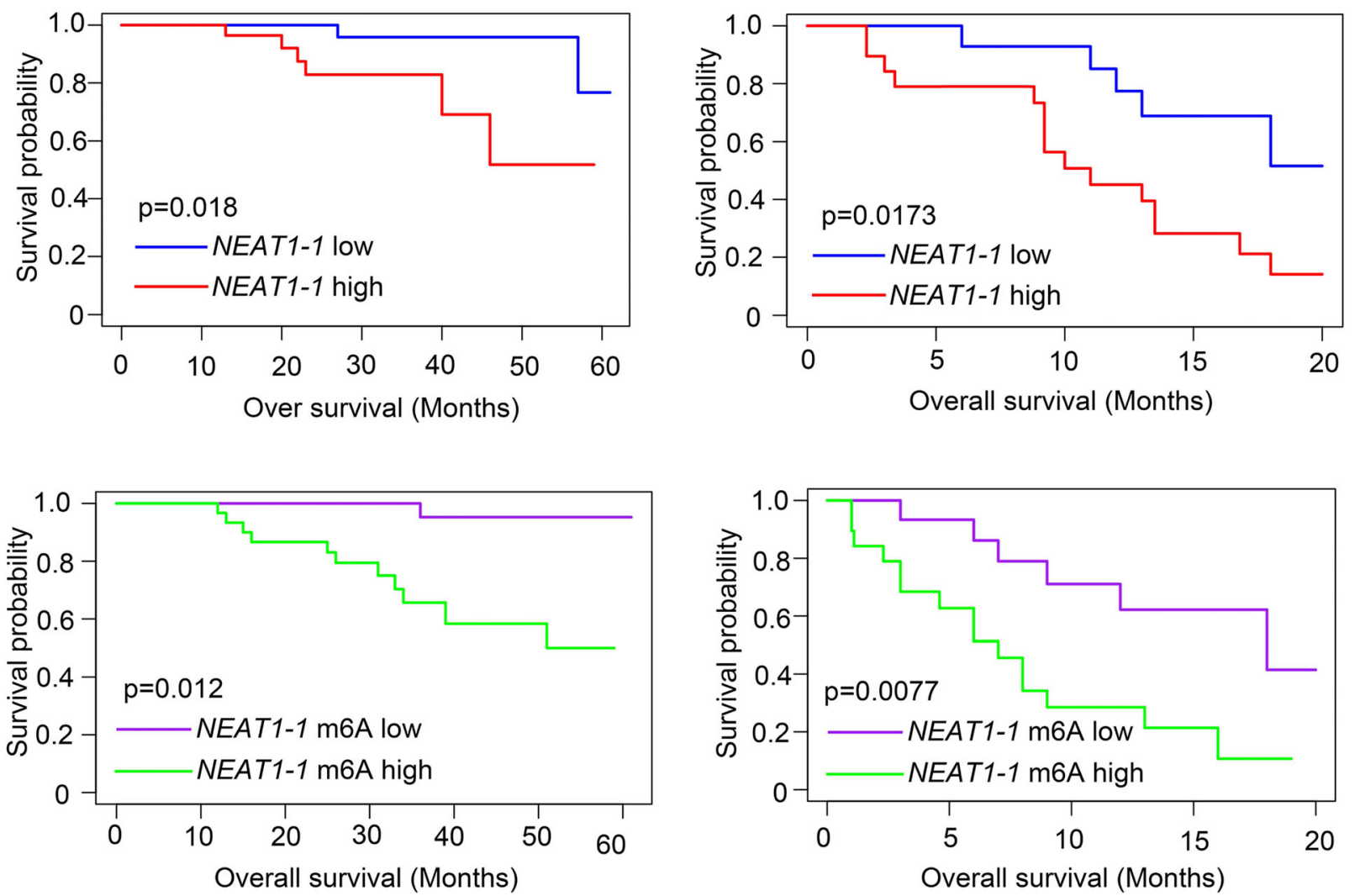

Fig. 1 m6A of NEAT1-1 was elevated in prostate cancer and was a negative prognostic factor for patients. a and $\mathbf{b}$ Box and whisker plot showing m6A signals upregulated in bone metastatic prostate cancer tissues. Analyswas of Second Xiangya Hospital (a) and Tianjin Medical University (b) data sets for levels of m6A and NEAT1-1 RNA were based on the m6A-RIP and RT-PCR. $n=30$ each group. $P$ values were shown in the figures. $\mathbf{c}$ and $\mathbf{d}$ Kaplan-Meier survival analyswas of the Tianjin Medical University data sets for the relationship between the levels of m6A of NEAT1-1, expression of NEAT1-1 and survival time in prostate cancers (c) and bone metastatic prostate cancer tissues (d). c, $\mathrm{n}=30$; $\mathrm{d}, n=20$. $P$ values were shown in the figures 
Table 1 The expressions of m6A on NEAT1 with clinic pathologic features

\begin{tabular}{|c|c|c|c|c|c|}
\hline \multirow[t]{2}{*}{ Characteristic } & & \multicolumn{2}{|c|}{ m6A level on NEAT1 } & \multicolumn{2}{|c|}{$P$-value } \\
\hline & & High & low & High & low \\
\hline \multirow[t]{2}{*}{ Age } & $<50$ & 15 & 7 & 0.315 & 0.276 \\
\hline & $\geq 50$ & 30 & 8 & & \\
\hline \multirow[t]{2}{*}{ Gleason score } & $\leq 6$ & 19 & 9 & 0.082 & 0.265 \\
\hline & $>6$ & 26 & 6 & & \\
\hline \multirow[t]{2}{*}{ Tumor stage } & $\mid+\|$ & 22 & 6 & 0.063 & 0.226 \\
\hline & $I I I+I V$ & 23 & 9 & & \\
\hline PSA recrutement (30 & No & 12 & 12 & 0.005 & 0.006 \\
\hline months) & yes & 33 & 3 & & \\
\hline \multirow[t]{2}{*}{ metastasis } & No & 10 & 13 & 0.001 & 0.001 \\
\hline & yes & 35 & 2 & & \\
\hline
\end{tabular}

METTL3 knocking-out P-18 cells (Fig. 2f). To further examine the m6A's role in interaction with CYCLINL1, we performed the RNA pull-down assay with WT and site\#4 m6A deletion mutated NEAT1-1. We revealed that only the \#4 m6A site mutation blocked the binding to CYCLINL1, but not \#1, \#2 and \#3 (Fig. 2g). Furthermore, Fig. $2 \mathrm{f}$ showed that site\#4 m6A mediated the NEAT1-1 interaction with CYCLINL1, but not ESR1, EZH2, and SUZ1. These data indicated that NEAT1-1 interacted with CYCLINL1 through its \#4 m6A site in prostate PDX primary cells.

\section{NEAT1-1 is a bridge to connect CYCLINL1 and CDK19}

Based on the mass spec data, we found that NEAT1-1 bound to CYCLINL1 and Cyclin-dependent kinase (CDK)19 (Fig. 2c). To demonstrate direct or indirect binding between CYCLINL1 or CDK19 and NEAT1-1, we searched the potential RNA binding residues (RBR) in the CYCLINL1 and CDK19. We used RBRDetector (http://ibi.hzau.edu.cn/rbrdetector) and found a GxxGxG domain in CDK19 and four arginine-rich domains in CYCLINL1, which were potential RNA recognized motifs (RRMs) (Fig. 3a and b). Additionally, there was no nature mutation in RRMs and known domains in CDK19 and CYCLINL1 (Fig. 3a and b). GxxGxG RNA binding domain ( $G$ is glycine, $x$ is any animo acid) is similar with the GxxGxG-domain in hnRNPU, which prefers to interact with U-rich RNA [48, 49]. The GxxGxG-domain is also a unique domain in CDK19, but not in its homolog CDK8 (Fig. S3b). GxxGxG domain deletion blocked the interaction with CDK19 and NEAT1-1 by in vitro transcribed RNA in P-18 cells (Fig. 3a). The arginine-rich domain is one of RNA recognized domains for RNA binding $[49,50]$. The two argininerich domains in the C-terminal of CYCLINL1 were required to interact with NEAT1-1 in P-18 cell lysate (Fig. $3 \mathrm{~b})$. To further determine the NEAT1-1 binding region in cell, we performed the interaction assay by individualnucleotide resolution CLIP (iCLIP). The iCLIP assay showed that NEAT1-1 bound to CDK19 inside 1-600 nt; and NEAT1-1 bound to CYCLINL1 inside 3100$3756 \mathrm{nt}$ (Fig. 3c and d). RNA electrophoretic mobility shift assay (EMSA) showed that CDK19 associated with the unique U-rich region (487-493 nt) in NEAT1-1 1$600 \mathrm{nt}$ (Fig. 3e), which is consistent with $\mathrm{CxxCxC}$ domain feature in hnRNPU binding [48]. RNA EMSA also showed that CYCLINL1 associated with $\mathrm{m6A}$ region inside NEAT1-13100-3756 nt (Fig. 3e and S3c). Coimmunoprecipitation (Co-IP) assay demonstrated that destroy of RNA inhibited the interaction between CYCL INL1 and CDK19 (Fig. 3f). Taken together, these data indicated that NEAT1-1 is a bridge to connect CYCL INL1 and CDK19.

Interestingly, CYCLINL1 is a bone specific expressed protein (Fig. S3d); CDK19 is a prostate specific expressed protein (Fig. S3d). We found that both of CYCLINL1 and CDK19 were highly expressed in bone metastatic prostate cancer tissues (Fig. S3e and f). These data suggest that the complex among CYCLINL1, CDK19 and NEAT1-1 may be a specific complex in bone metastatic prostate cancer.

\section{NEAT1-1 activates pol II Ser2 phosphorylation through $\mathrm{m} 6 \mathrm{~A}$ in vitro and in prostate cancer}

CDK19 is a homolog of CDK8, and they are cyclindependent kinase that regulates RNA Polymerase II (RNPII) to mediate gene expression [51-53]. CDK8 with its partner CYCLINC phosphorylates the CTD of RNPII in vitro and in vivo [51]. However, the molecular detail for CDK19 regulation is limited. In this study, we found CDK19 can be pulled down by NEAT1-1 in P-18 (Fig. 2c). To examine the activity of CDK19, NEAT1-1 and CYCLINL1 complex, we consequently translated the CDK19 or CYCLINL1 in vitro using TNT quick coupled transcription/translation system kit and specific magnetic beads. Before the kinase assay in vitro, we determined the m6A of NEAT1-1 in vitro. The dot blot assay showed that NEAT1-1 RNA can be methylated in P-18 cell, but not in METTL3-KO cells (Fig. S4a). Then we purified the NEAT1-1 from these cells by biotin labeled probes. In vitro RNPII phosphorylation assay showed that adding NEAT1-1 activated the RNPII phosphorylation in Ser-2 (Fig. 4a and S4a). Next, we found that NEAT1-1 binding domain-deletion in CYCLINL1 blocked the RNPII Ser2 phosphorylation induced by CDK19 and CYCLINL1 in vitro (Fig. S4b). However, the kinase activity of complex formed by CDK19 and CYCL INC was not blocked by NEAT1-1 binding domaindeletion (Fig. S4b). These data suggest that the kinase activity of complex formed by CDK19 and CYCLINL1 required NEAT1-1 interaction. It was noticed that most 
a
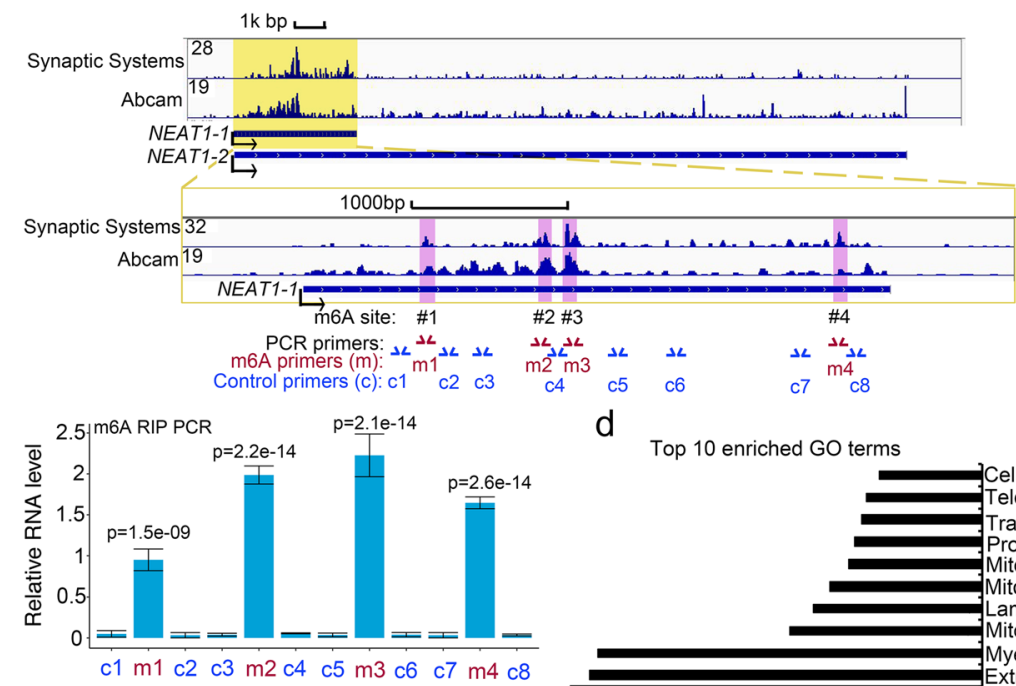

d

NEAT1-1 biotin purification

\begin{tabular}{lll}
\hline $\begin{array}{l}\text { Purificated } \\
\text { protein }\end{array}$ & $\begin{array}{l}\text { Control } \\
\text { peptides }\end{array}$ & $\begin{array}{l}\text { METTL3-KD } \\
\text { peptides }\end{array}$ \\
\hline
\end{tabular}

\begin{tabular}{lrr}
\hline ERa & 6 & 5 \\
hnRNPK & 9 & 6 \\
EZH2 & 16 & 18 \\
SUZ12 & 8 & 9 \\
CYCLINL1 & 19 & 1 \\
CDK19 & 12 & 11 \\
\hline
\end{tabular}

Top 10 enriched GO terms

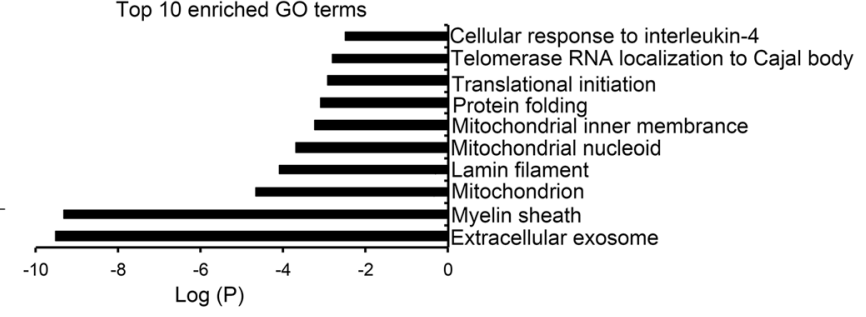

Top 10 enriched KEGG terms

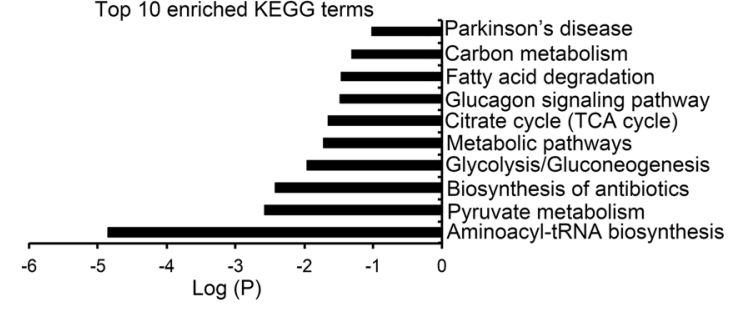

e Probe $f$
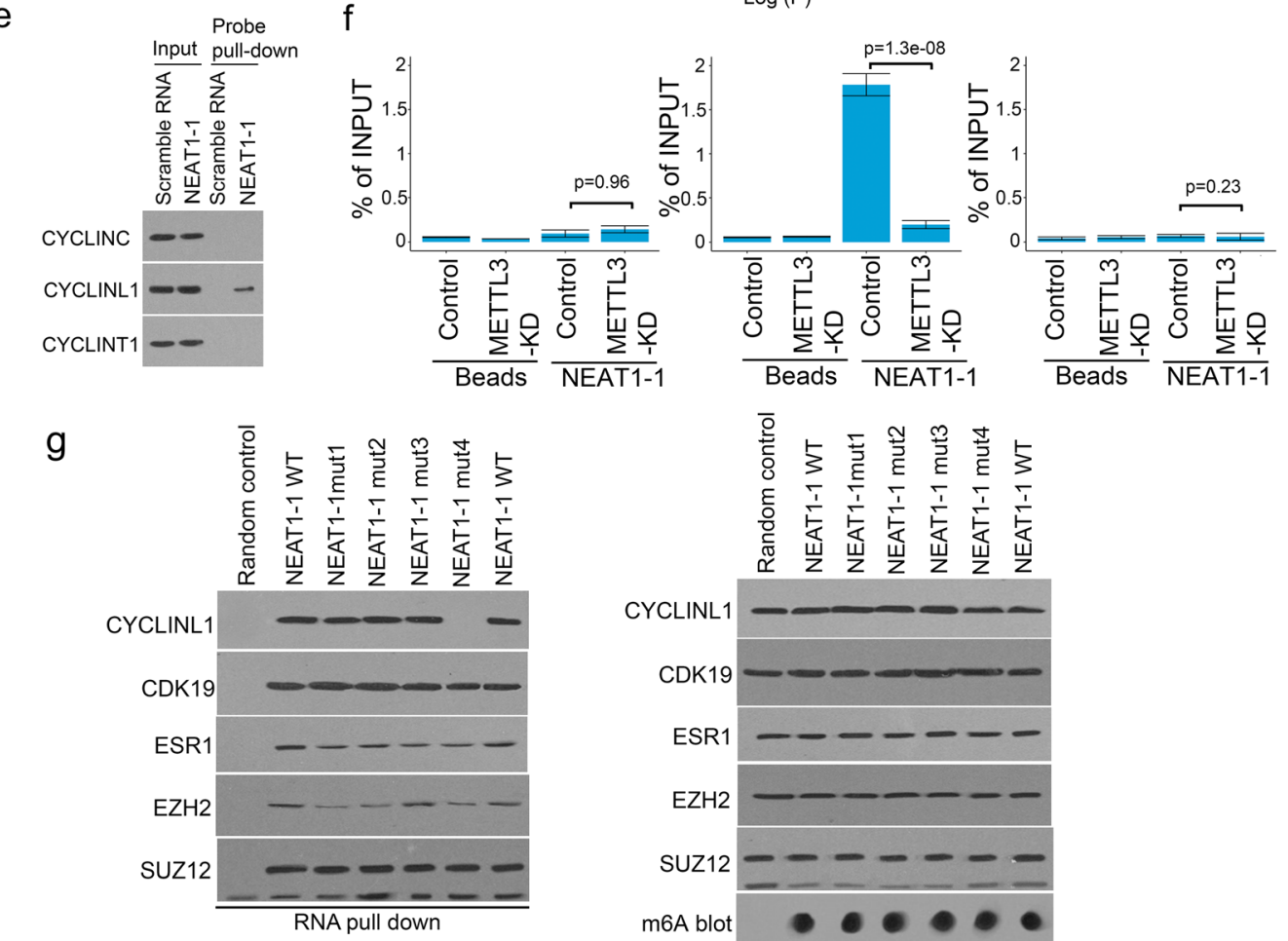

T1-1
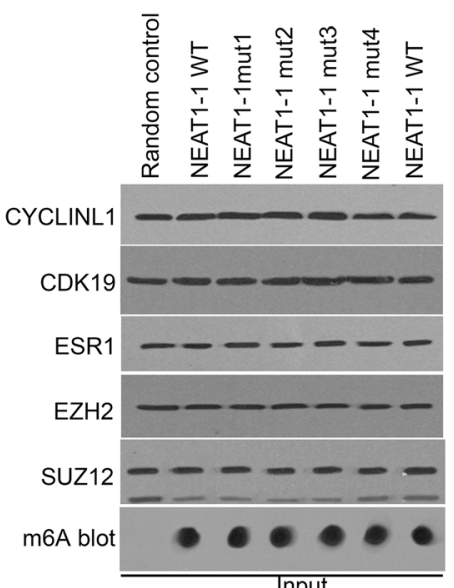

Fig. 2 (See legend on next page.) 
(See figure on previous page.)

Fig. 2 m6A sites were identified in NEAT1-1 in prostate cancer. a m6A RIP-seq analyswas of m6A sites of NEAT1 by two independent antibodies. The m6A profiles of NEAT1 were shown in genome browser. $\mathbf{b}$ Quantitative PCR verification of m6A RIP results on the gene body of NEAT1-1 with m6A antibody against the m6A sites in P-18 primary cells. Means and standard deviations (error bar) were determined from three replicates. Error bars represent mean \pm SD for triplicate experiments. $P$ values were shown in the figures. $\mathbf{c}$ The top hits of NEAT1-1 pull-down proteins were identified by TAP-MS. The numbers of peptides were indicated in the Iwast. $\mathbf{d}$ Ontology analyswas were by DAVID (david.ncifcrf.gov) and KEGG analyswas by DAVID and KAAS (www.genome.jp/kass-bin). e Biotin pull-down assay by incubating biotin-labeled specific probes targeting NEAT11 with P-18 cell lysate followed by Western blot with CYCLINC1, CYCLINL1 and CYCLINT1 antibodies. f CLIP-gPCR analyswas of CYCLINL1 binding at the NEAT1-1 in P-18 control or METTL3 knocking out cells transfected with control or METTL3-specific sgRNA. Immunoprecipitated RNAs were detected by real-time PCR. All data shown were mean values \pm SD (error bar) from three replicates. $P$ values were shown in the figures. $\mathbf{g}$ NEAT11 pull-down assay use biotin-labeled NEAT1-1 and NEAT1-1 mutants (mut). The RNA and protein complexes were detected by western blot

of NEAT1-1 are located in nucleus [54-56]. We checked NEAT1-1's location in P-18 cells, the data showed that transfected NEAT1-1 was located in nucleus in P-18 cell (Fig. S4c), suggesting the NEAT1-1 may take nuclear function in prostate cancer cell. We demonstrated that knocking out NEAT1-1 in P-18 by siRNAs and ASOs deterred the RNPII phosphorylation in Ser-2, but not Ser-5 (Fig. 4b). In addition, we found that RNPII Ser2 phosphorylation was downregulated by depletion of NEAT1-1, but not depletion of MALAT1, Xist or ARLNC1 (Fig. 4c and S4d). To further determine the exact the m6A site, we transfected the \#4 sitemutation (3494A deletion) NEAT1-1 into P-18 cells with NEAT1-1 homogeneous knocking out (KO) by CRISPR system. We confirmed that transcribed NEAT1-1 increased the m6A-modified NEAT1-1 level in P-18 NEAT1-1-KO cells (Fig. S4e) and also confirmed the homogeneous NEAT1-1 KO efficacy and ruled out the off-target effect (Fig. S5a-c). We found that NEAT1-1 WT increased the RNPII Ser-2 phosphorylation, but \#4 mutation failed to do it (Fig. 4d). These data indicated that NEAT1-1 activated RNPII Ser-2 phosphorylation through \#4 m6A site on NEAT1-1.

\section{NEAT1-1 recruites CYCLINL1 and CDK19 on RUNX2 promoter via $\mathrm{m6A}$ site \#3}

To search the target genes of CYCLINL1/CDK19/ NEAT1-1 complex, we performed RNA-seq to find the regulated genes in two groups. One group was ASO control vs. NEAT1-1 KD group; another group was P-18 NEAT1-1 KO cells transfected with NEAT1-1 WT vs. NEAT1-1 site\#4-m6A deletion. The venn diagram data showed that 5166 genes were the target genes that can be regulated by both depletion of NEAT1-1 and deletion of NEAT1-1 site\#4 m6A (Fig. 5a, Table S3 and Table $\mathrm{S} 4)$. Some target genes were bone metastasis related genes, such as RUNX2, EPHA3 and ALDH3A2 [57-59]. We confirmed the RNA levels' changes in NEAT1-1 WT and site\#4-m6A deletion cells by RT-PCR (Fig. S5d). RUNX2 is a central driver in bone metastatic prostate cancer [58, 60-62]. To further search the target gene of complex of NEAT1-1, CYCLINL1 and CDK19, we hypothesis that the complex select its target through
RNA-DNA interaction. We predicted the potential target gene by DNATriplex software (http://ncrna.smu. edu.cn/show/DNATriplex). The prediction data showed that NEAT1-1 may interacted with the promoter of RUNX2, which is vital marker and driver in bone metastatic prostate cancer (Fig. 5b). To confirm this prediction, we performed the chromatin isolation by RNA purification (ChIRP) in P-18. The ChIRP data revealed that NEAT1-1 interacted with the promoter of RUNX2 in $-200 \mathrm{nt} \sim-50 \mathrm{nt}$ region (Fig. 5c), consistent with software prediction result. To know whether NEAT1-1 recruited CYCLINL1 or CDK19 onto promoter of RUNX2, we tested the binding of CYCLINL1 by chromatin immunoprecipitation (ChIP) assay. ChIP data showed that depletion of NEAT1-1 by CRISPR system and rescued with NEAT1-1 \#4 m6A-mut blocked the association of CYCLINL1 with promoter of RUNX2 (Fig. $5 \mathrm{~d})$. Furthermore, overexpression of NEAT1-1 WT increased the RUNX2 RNA and protein levels, but NEAT1-1 m6A \#4-mutation failed to upregulate RUNX2 RNA in P-18 cells and P-34 cells and protein levels in P18 cells (Fig. 5e and f). Taken together, these data \#4 m6A site facilities NEAT1-1 recruit CYCLINL1 and CDK9 onto promoter of RUNX2 through RNA-DNA interaction.

\section{NEAT1-1 enhanced prostate PDX growth through m6A}

To test the m6A of NEAT1-1 function in vivo, we used the xenograft model to determine what extent m6A regulation of NEAT1-1 contributes to prostate PDXs growth and metastasis. The mice tail vein injection data showed that overexpression of NEAT1-1 WT decrease the mice survival span, and also increase the metastatic probability to pelvis bone and/or lung (Fig. 6a, b and S6a). Furthermore, NEAT1-1 WT promoted flank tumor growth of P-18 and P-34 PDXs, but not NEAT11 \#4 m6A-mutant (Fig. 6c and d). The RT-PCR data in each xenograft sample showed that NEAT1-1 WT and m6A-mut groups had higher levels of NEAT1-1 RNAs than vehicle groups of P-18 cell and P-34 cell xenografts. However the level of NEAT1-1 was no significant difference among the xenografts in NEAT1-1 WT and m6Amut overexpression groups (Fig. S6b). RUNX2 RNA 
a

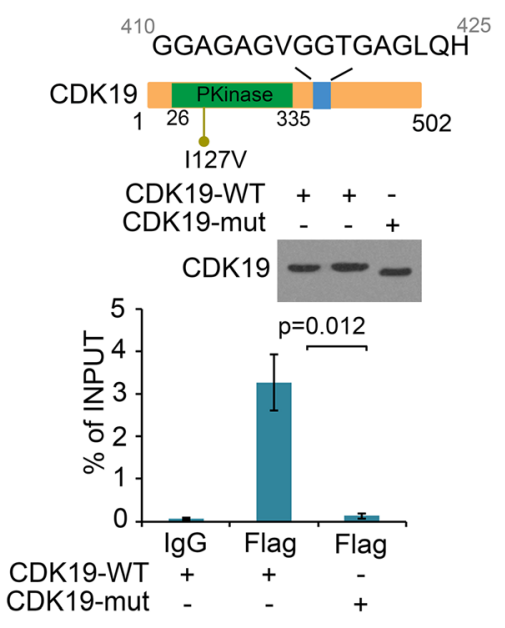

b mut4: 521HGRHRR 526

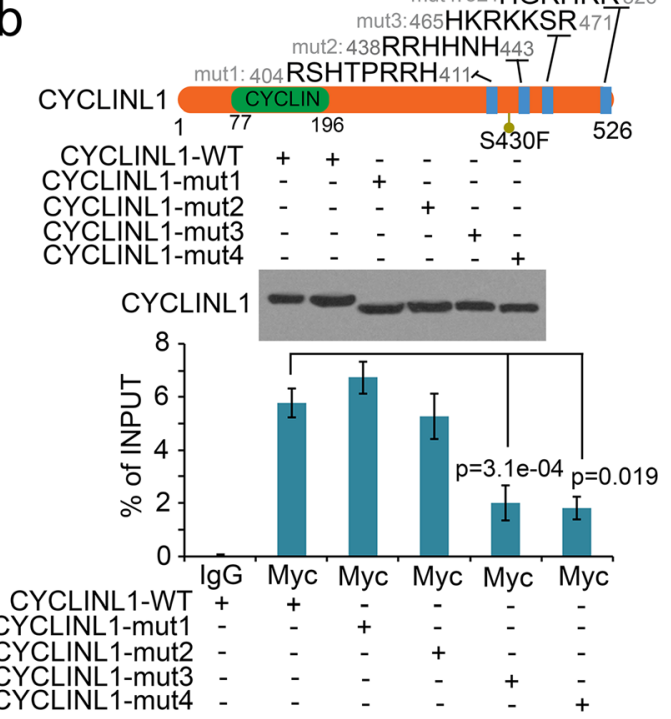

C

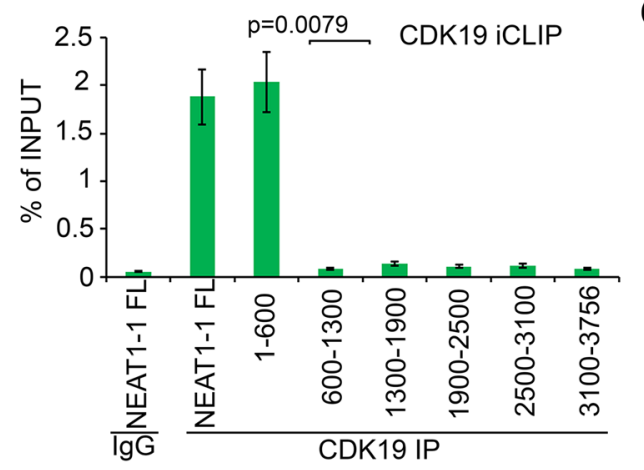

d

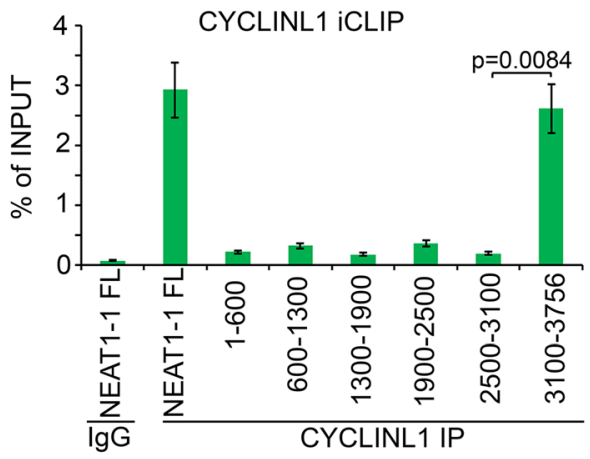

e

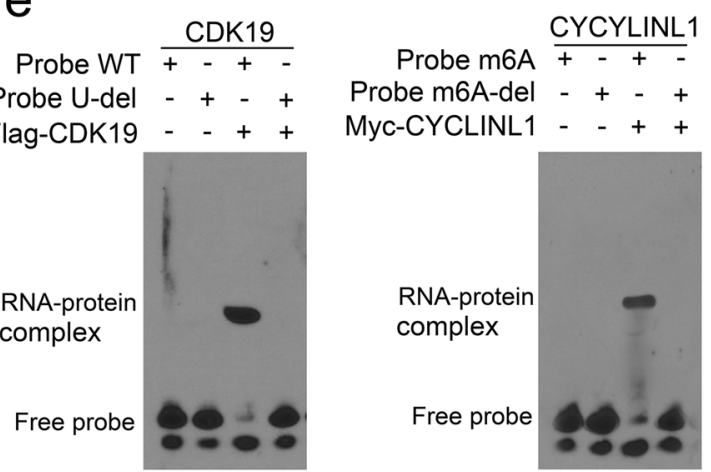

f

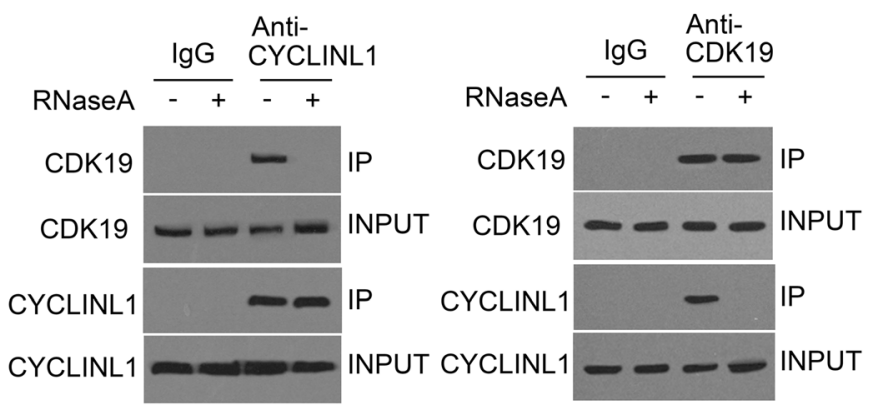

Fig. 3 NEAT1-1 interacted with CYCLINL1 and CDK19 through m6A site \#4. a and b Upper, protein diagrams with RNA recognition motif (rrm) were shown. Lower, CLIP-qPCR analyswas of Flag or Myc binding at the NEAT1 in P-18 out cells transfected with Flag-CDK19-WT, Flag-CDK19-mut (rrm deletion), Myc-CYCLINL1-WT or Myc-CYCLINL1-muts (rrms deletion). Nature mutations were shown in the diagrams. PKinase, protein kinase domain. All data shown were mean values \pm SD (error bar) from three replicates. $P$ values were shown in the figures. $\mathbf{c}$ and $\mathbf{d} C L I P-q P C R$ analyswas of CDK19 or CYCLINL1 binding at the NEAT1-1 in P-18 cells with different primers targeting different regions. e RNA EMSA evaluation of CDK19 or CYCLINL1 binding of NEAT1-1 RNA probes. Flag-CDK19 or MyC-CYCLINL1 recombinant proteins were produced using Quick coupled transcription/translation kit through T7 promoter in vitro. Flag-CDK19 or Myc-CYCLINL1 recombinant proteins were incubated with biotin-labeled in vitro transcribed NEAT1-1 probes in the presence or absence of m6A modification (single nucleotide "A" deletion (3494 nt of NEAT1-1)), followed by PAGE and immune blotting with HRP-conjugated streptavidin. $\mathbf{f}$ Co-IP of endogenous CYCLINL1 or CDK19 with CDK19 or CYCLINL1 from P-18 cell lysate pre-treated with RNaseA in $37^{\circ} \mathrm{C}$ for $30 \mathrm{~min}$. The protein complexes were detected by western blot 


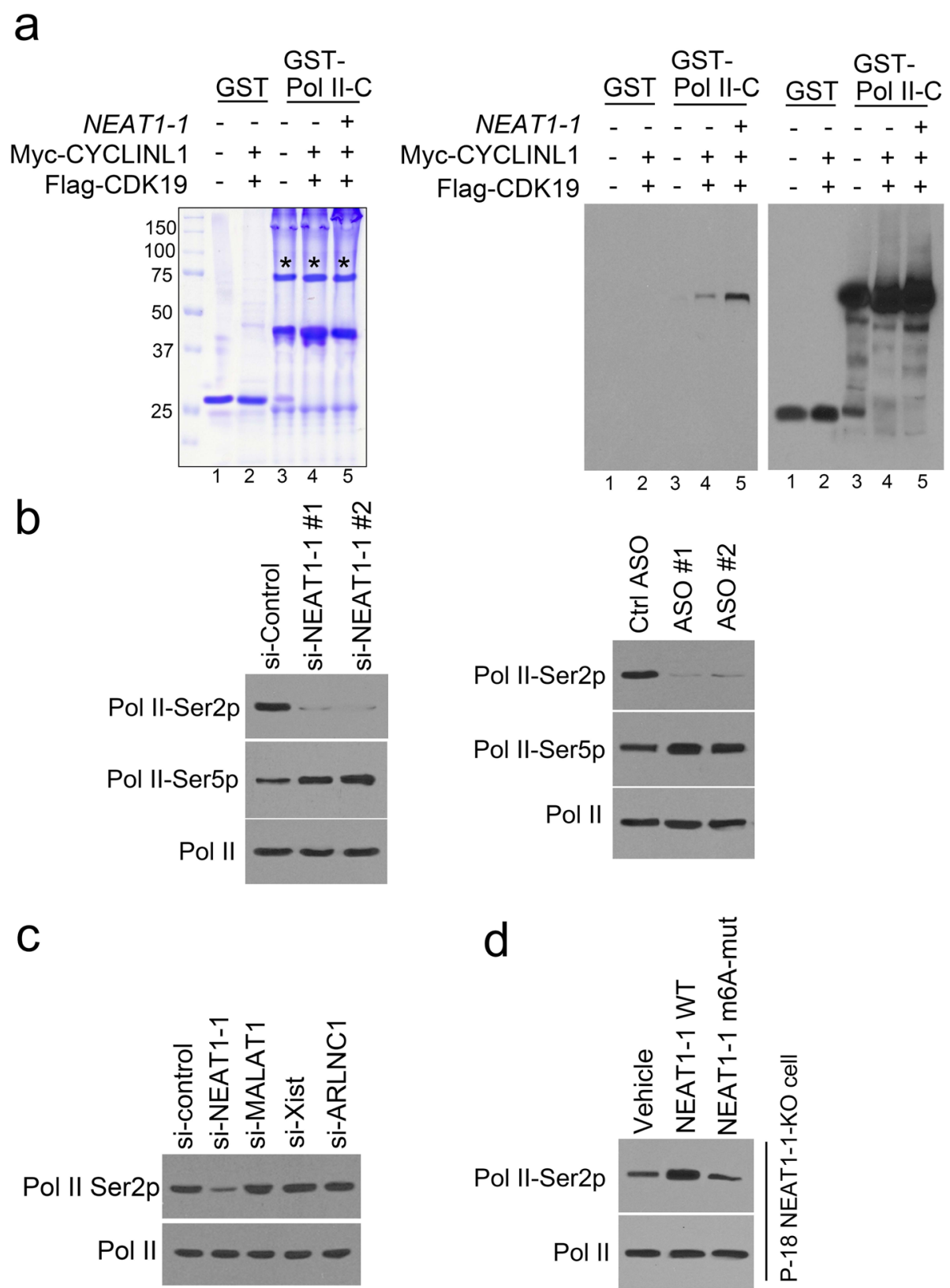

Fig. 4 NEAT1-1 activated Pol II Ser2 phosphorylation through m6A in vitro and in prostate cancer. a Left, Coomassie blue staining of GST and GST-Pol II-C terminal domain (GST-Pol II-C, 372 amino acids of the Pol II-C terminal domain) recombinant proteins used for kinase assay. A star indicate the GST and GST-Pol II-C recombinant protein bands. Right, determination of CTD kinase (CDK19) activity. $1 \mu$ g GST or GST-Pol II-C terminal fusion proteins (in all lanes) and $2 \mu \mathrm{g}$ NEAT1-1 RNA (lane 5) were incubated with CDK19 (lane 2,4,5) and CYCLINL1 produced from Quick coupled transcription/translation kit. After extensive washing, GST-beads were collected and subject to SDS/PAGE and western blot with Pol II Ser-2p antibodies. $\mathbf{b}$ Expression of Pol II Ser-2, Sre-5 phosphorylations and total Pol II proteins were measured by western blot in P-18 cells infected with control or NEAT1-specific siRNAs or in transfected with control or PSA eRNA-specific ASOs. c Expression of Pol II Ser-2 phosphorylations and total Pol II proteins were measured by western blot in P-18 cells with control siRNAs and siRNA pool of NEAT1, MALAT1, Xwast or ARLNC1. $\mathbf{d}$ Expression of Pol II Ser-2 phosphorylations and total Pol II proteins were measured by western blot in P-18 NEAT1-1-KO cells infected with control or NEAT1-1-overexpressed plasmids or NEAT1-1-m6A-mutants plasmids

levels were significantly higher in NEAT1-1 WToverexpressed flank tumors than m6A-mut overexpressed tumors (Fig. 6e). Protein levels of RUNX2 were also higher in NEAT1-1 WT-overexpressed flank tumors than m6A-mut overexpresed tumors (Fig. 6f). The data indicated that NEAT1-1 WT upregulated the phosphorylation of RNPII Ser2 and RUNX2 RNA and protein levels compared to vehicle group, but the m6A 
a

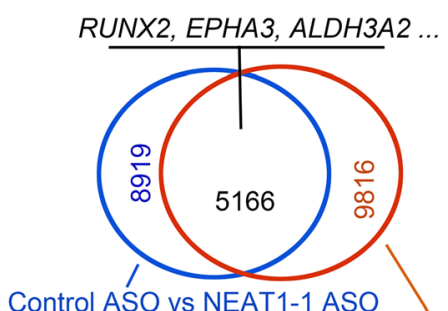

b

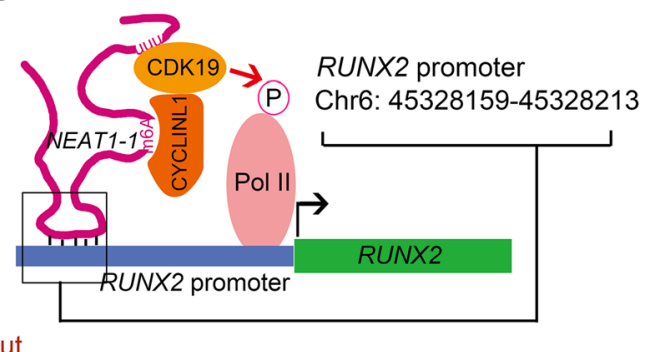

C

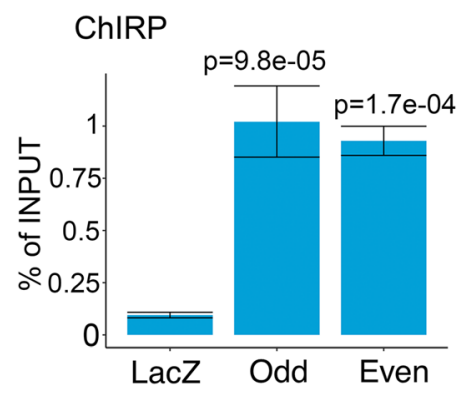

e RT-PCR $\mathrm{p}=6.8 \mathrm{e}-09 \quad$ RUNX2

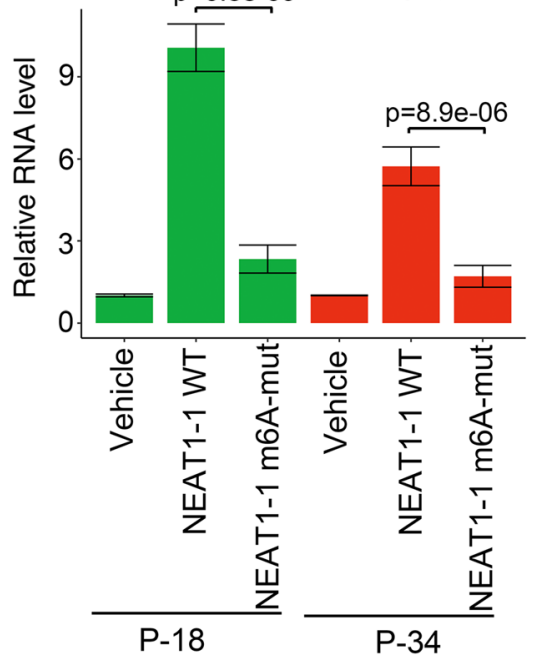

d

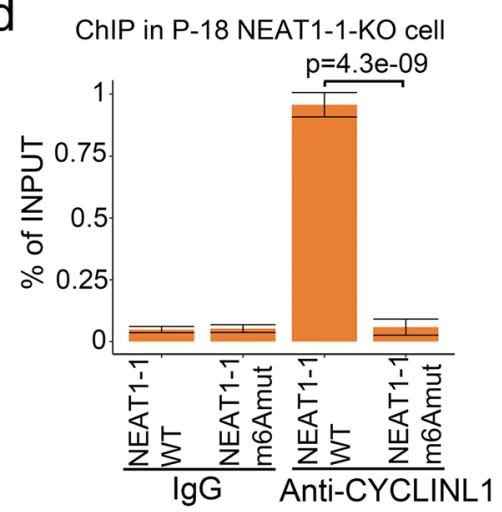

f

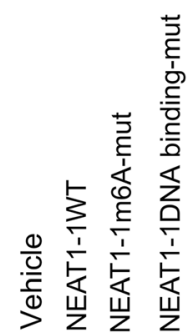

Pol II-Ser2p - $-\infty$

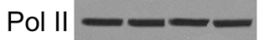

RUNX2 - - -

Tubulin - - -

Fig. 5 NEAT1-1 recruited CYCLINL1 and CDK19 on RUNX2 promoter via m6A site \#4. a Venn diagram showing that genes mediated by NEAT1-1 knocking down overlapped with genes mediated by NEAT1-1 site\#4 m6A deletion (nt3494A deletion) in P-18 cells ( $P=9.3 \mathrm{e}-10$, permutation test). b A diagram of CDK19 and CYCLINL1 interaction through NEAT1 on promoter of target gene. c ChIRP assay using biotin-labeled LacZ or NEAT11-specific DNA probes and streptavidin beads. Pulldown DNA was analyzed by real-time PCR. All data shown were mean values \pm SD (error bar) from three replicates. $P$ values were shown in the figures. $\mathbf{d}$ CHART-qPCR analyswas of CYCLINL1 binding at the NEAT1-1 WT and m6A-mut in P18 NEAT1-KO cells. Immunoprecipitated RNAs were detected by real-time PCR. All data shown were mean values \pm SD (error bar) from three replicates. m6A-mut was site\#4 m6A deletion in NEAT-1. $P$ values were shown in the figures. e RUNX2 expressions were measured by qRT-PCR in P-18 and P-34 primary cells. Means and standard deviations (error bar) were determined from three replicates. Error bars represent mean \pm SD for triplicate experiments. m6A-mut was site\#4 m6A deletion in NEAT-1. P values were shown in the figures. $\mathbf{f}$ Expression of RUNX2, Tubulin, Pol II Ser-2 phosphorylations and total Pol II proteins were measured by western blot in P-18 NEAT1-1-KO cells infected with control or NEAT1-1-WT, NEAT1-1-m6A-mutant (nt3494A deletion) or NEAT1-1 promoter-binding mutant (nt874-899 deletion) 


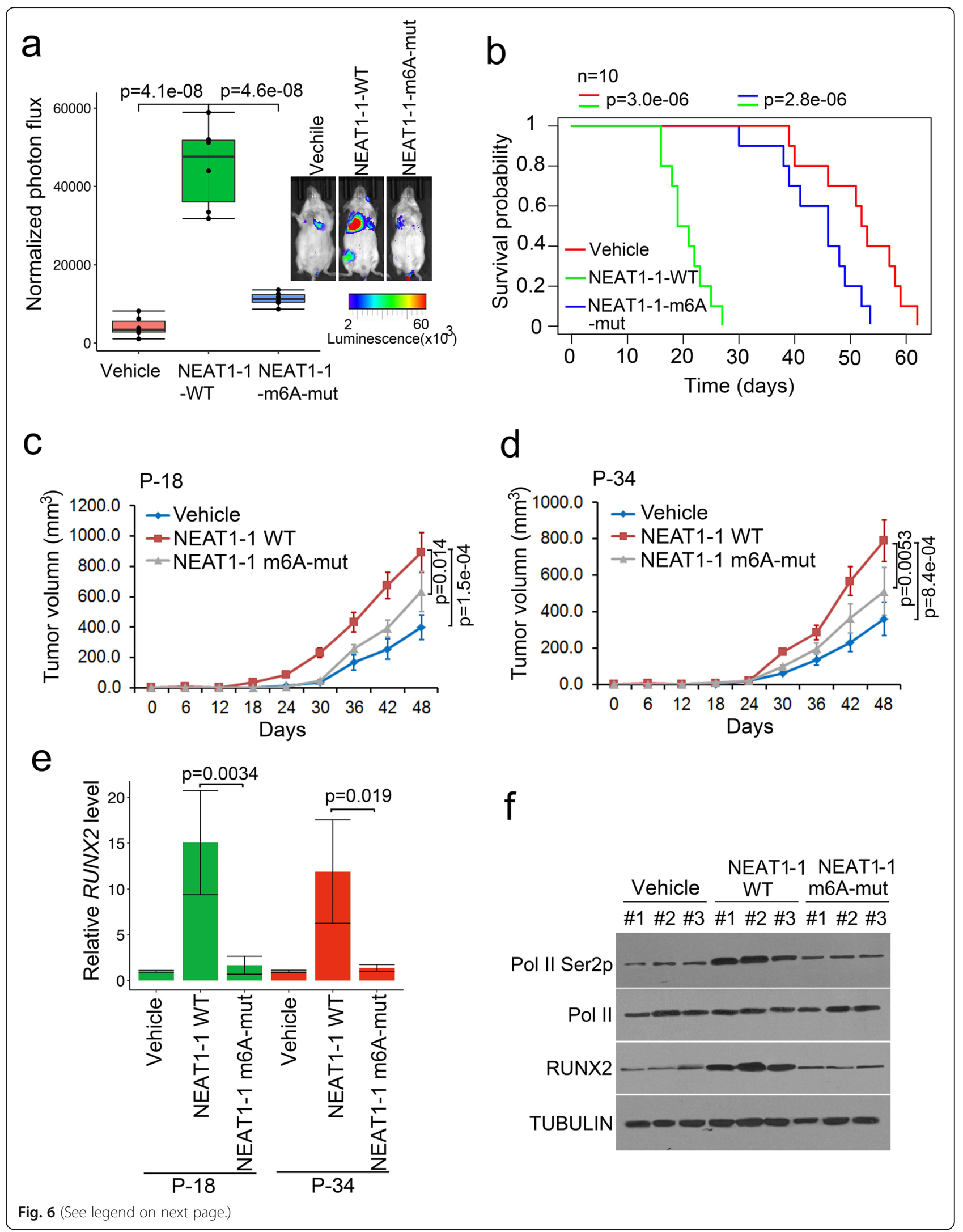


(See figure on previous page.)

Fig. 6 NEAT1-1 enhanced prostate PDX metastaswas and growth through m6A. a Luciferase-expressing P-18 cells $\left(3 \times 10^{6}\right)$ transfected with lentivirus were injected through tail vein into NSG mice ( $n=10$ each group). The mice were subjected to bioluminescent imaging and bioluminescent signals were quantified. Bioluminescent flux (photons $/ \mathrm{s} / \mathrm{sr} / \mathrm{cm}^{2}$ ) was determined for lesions in mice, the ends of the box were the upper and lower quartiles and the box spans the interqurtile range; the median was marked by a vertical line inside the box; the whiskers were the two lines outside the box that extend to the highest and lowest observations. $P$ values were shown in the figures. Representative images from 10 different samples were shown. $\mathbf{b}$ Kaplan-Meier survival analyswas of the mice data for the survival time in three groups. Three groups were indicated in the figure. $\mathbf{c}$ and $\mathbf{d}$ Effect of m6A of NEAT1 on growth of bone metastatic prostate cancer xenografts. $5 \times 10^{6} \mathrm{P}-18$ and P-34 cells were injected into NSG mice ( $n=6$ each group). The tumor growth was measured every 6 days for 48 days, and the data were shown in the bottom panel. Data shown as means \pm SD $(n=6)$. Statwastical significance was determined by two-tail Student's $t$-test. $P$ values were shown in the figures. e and $\mathbf{f}$ RUNX2 expressions were measured by qRT-PCR and western blot in P-18 and P-34 xenograft tissues. Means and standard deviations (error bar) were determined from three replicates. Error bars represent mean \pm SD for triplicate experiments. $P$ values were shown in the figures. Expression of RUNX2, Tubulin, Pol II Ser-2 phosphorylations and total Pol II proteins were measured by western blot in P-18 three random xenograft tissues

mutation group failed to do these (Fig. 6e and f). Therefore, the data demonstrated that m6A of NEAT1-1 elevated the tumor growth and metastasis in mice PDX model.

\section{Discussion}

m6A in mammalian was identified as a highly abundant modification of mRNA several decades ago [7-9]. The writers, erasers and readers of m6A can add, remove and recognize the m6A site in mRNA and do the function including: nuclear transport, splicing, stability and translation [10-12, 14]. In long noncoding RNA, m6A was found on metastasis-associated lung adenocarcinoma transcript-1 (MALAT1) [63] and X inactive-specific transcript (Xist) [64]. In this study, we found a new m6A modified long noncoding RNA - NEAT1-1. The m6A level of NEAT1-1 was positively related to prostate cancer progression, bone metastasis and negatively related to patients' survival (Fig. 1). It indicated that m6A in NEAT1-1 may exhibit oncogenic function in cancer progression. m6A in MALAT1 associates with HNRNPC to affect target gene expression [63] and m6A in Xist binds to YTHDC1 to mediate the transcript silencing [64]. Here, we found a new m6A reader - CYCLINL1. CYCL INL1 is a bone marrow specific protein [65]. Interestingly, we found that CYCLINL1 also expressed in prostate cultured primary cell and PDXs from bone metastatic patients. CYCLINL1 bound to NEAT1-1 m6A site with its R-rich C-terminal domain. High m6A level NEAT1-1 activated CYCLINL1 and formed a new complex with CDK19 which was specific high expressed in prostate tissues. Thus, NEAT1-1 induced the activity of CYCLINL1/CDK19 complex, and recruited it onto RUNX2 promoter through RNA/DNA hybridization. Therefore, we identified the new m6A function in long noncoding RNA and pushed prostate cancer progression going ahead.

Prostate cancer is the second-most common cause of cancer death in men of US $[1,2]$. The main mortality cause of prostate cancer is metastasis and about $80 \%$ of the metastatic prostate cancer cells spread to bones [3, 4]. The reason why prostate cancer settling in bone "soil" is limited to know. In mechanism, how prostate cancer express bone related genes and synchronize itself to be adapted to bone environment is also an interesting question. There were several key proteins found in in bone and bone metastatic prostate cancer, including RUNX2 [58, 60, 61]. Interestingly, CYCLINL1 is a bone specific expressed protein; CDK19 is a prostate specific expressed protein (Fig. S3). We found that both of CYCLINL1 and CDK19 were highly expressed in bone metastatic prostate cancer tissues. These data suggest that the complex from CYCLINL1, CDK19 and NEAT11 might be a specific complex in bone metastatic prostate cancer. We also found that the high activation of CYCLINL1 was related to high level of m6A in NEAT11. NEAT1-1 activated the CYCLINL1 through the m6A site interaction with CYCLINL1. Depletion of NEAT1-1 or mutation in the m6A of NEAT1-1 inhibited the CYCLINL1 activity and RUNX2 RNA levels, which was the downstream target of CYCLINL1 and CDK19 in cell and xenograft (Fig. 6). With the RUNX2 and related pathway, bone metastatic prostate cancer exhibited the similar features with bone and can be survival in the environment of bone.

Taken together, we found the NEAT1-1 m6A facilitated the oncogenic function of new complex CYCL INL1/CDK19 in bone metastatic prostate PDXs. The findings indicate that ncRNA m6A have vital role in regulating prostate cancer progression and may be novel target for cancer therapy and diagnosis.

\section{Conclusions}

In conclusion, we identified the novel m6A function in ncRNA that played an oncogenic role in bone metastatic prostate cancer and was correlated with poor prognosis. Further experiments demonstrated that NEAT1-1 m6A facilitated the oncogenic function of new complex CYCL INL1/CDK19 for Pol II Ser2 phosphorylation. Our results revealed that ncRNA $\mathrm{m} 6 \mathrm{~A}$ had vital role in 
regulating prostate cancer progression and may be novel target for cancer therapy and diagnosis. The regulatory network involving the new complex CYCLINL1/CDK19/ NEAT1-1 might provide new insight into the potential mechanism of the pathogenesis and development of bone metastatic prostate cancer.

\section{Supplementary Information}

The online version contains supplementary material available at https://doi. org/10.1186/s12943-020-01293-4.

Additional file 1: Supplemental Fig. 1. m6A of NEAT1-1 was elevated in prostate cancer and was a negative prognostic factor for patients. (a) Box and whisker plot showing NEAT1-1 RNA signals upregulated in prostate cancer tissues compwered to normal tissues in TCGA data set. Data from GEPIA. (b) Box and whisker plot showing NEAT1 m6A signals upregulated in bone metastatic prostate cancer tissues. Analyswas of Tianjin Medical University data sets with fresh samples for levels of m6A and NEAT1 RNA were based on the m6A-RIP and RT-PCR. $n=30$ each group. $P$ values were shown in the figures. (c) Kaplan-Meier survival anaIyswas of the TCGA data set for the relationship between the levels of NEAT, expression of NEAT1-1 and survival time in prostate cancers. Data from GEPIA. Supplemental Fig. 2. m6A sites and secondary structure in NEAT1. (a) m6A RIP-seq analyswas of m6A sites of FRMD8 by two independent antibodies. The m6A profiles of FRMD8 were shown in genome browser. (b) NEAT1-1 and NEAT1-2 expressions were measured by qRTPCR in P-18 primary cells. M6A levels of NEAT1-1 and NEAT1-2 were measured by m6A-RIP-PCR in P-18 primary cells. Means and standard deviations (error bar) were determined from three replicates. Error bars represent mean \pm SD for triplicate experiments. $P$ values were shown in the figures. (c-f) Secondary structure of NEAT1-1 predicted by https://rna. tbi.univie.ac.at. m6A putative motif and sequences were shown in each figures. (g) NEAT1-1 expressions were measured by qRT-PCR in 293 T and P-18 primary cells. Transfected NEAT1-1 expressions were measured by qRT-PCR using primers targeting NEAT1-1 and plasmid in P-18 primary cells. Means and standard deviations (error bar) were determined from three replicates. Error bars represent mean \pm SD for triplicate experiments. $P$ values were shown in the figures. Supplemental Fig. 3. CYCLINL1 and CDK19 in tissues. (a) Expression of METTL3 and Vinculin proteins were measured by western blot in P-18 METTL3-KO cells infected with control or METTL3 KO CRWASPR-Cas9 plasmids. (b) Amino acid sequence comparwason of CDK8 and CDK19. (c) Dot blot of m6A and biotin to testing NEAT1-1 WT and m6A-deletion probes using anti-m6A and anti-biotin antibodies. $0.5 \mu \mathrm{g}$ RNA for each dot. (d) CYCLINL1 and CDK19 RNA levels in normal human tissues. Data from GTEx. (e and f) Box and whisker plot showing CYCLINL1 and CDK19 signals upregulated in bone metastatic prostate cancer tissues. Analyswas of Tianjin Medical University data sets for levels of CYCLINL1 and CDK19 RNA were based on the RT-PCR. $n=30$ each group. Supplemental Fig. 4. NEAT1-1 interacted with CYCLINL1 and CDK19. (a) Left panel, dot blot of m6A to testing NEAT1-1 full length RNA using anti-m6A. $0.5 \mu \mathrm{g}$ RNA for each dot. Methylene blue was used to measure input loading. Right panel, $1 \mu \mathrm{g}$ GST or GST-Pol II-C terminal fusion proteins and $2 \mu \mathrm{g}$ NEAT1-1 RNA were incubated with CDK19 and CYCLINL1 produced from Quick coupled transcription/translation kit. After extensive washing, GST-beads were collected and subject to SDS/PAGE and western blot with Pol II Ser-2p, total Pol II and GST antibodies. (b) GST or GST-Pol II-C terminal fusion proteins and $2 \mu \mathrm{g}$ NEAT1-1 RNA were incubated with CDK19 and CYCLINL1 WT or mutated proteins produced from Quick coupled transcription/translation kit from T7 promoter. After extensive washing, GST-beads were collected and subject to SDS/PAGE and western blot with Flag, Myc, Pol II Ser-2p, total Pol II and GST antibodies. (c) NEAT1-1 RNA (green) and AR RNA (red) were stained using probes with FITC or TEXAS-RED dye. Scale bar was $10 \mu \mathrm{m}$. (d) NEAT1-1, MALAT1, Xist and ARLNC1 expressions were measured by GRT-PCR in P-18 primary cells. Means and standard deviations (error bar) were determined from three replicates. Error bars represent mean \pm SD for triplicate experiments. $P$ values were shown in the figures. (e) NEAT1-1 site\#4 m6A levels were measured by m6A RIP PCR using primers targeting site\#4 in P-18 primary cells. Means and standard deviations (error bar) were determined from three replicates. Error bars represent mean \pm SD for triplicate experiments. $P$ values were shown in the figures. Supplemental Fig. 5. Sanger sequence showed NEAT1-1 knocking out in P-18 cells. (a) gRNAs sequences were shown in the two sides of NEAT1-1 RNA in genome. (b) NEAT1-1 full length and knocking out PCR products were amplified using primers outside the NEAT1-1. (c) gRNAs sequences were shown in the possible target in chromatin 22. (d) Target genes' expressions were measured by qRT-PCR in P-18 NEAT1-1 KO cells transfected with NEAT1-1 WT and NEAT1-1 m6A-mut. Means and standard deviations (error bar) were determined from three replicates. Error bars represent mean \pm SD for triplicate experiments. $P$ values were shown in the figures. Supplemental Fig. 6. X-ray for detection of mice bone. (a) Mice were scanned using a microcomputed $x$-ray system. Representative images from 10 different samples were shown. (b) NEAT1-1 expressions were measured by qRTPCR in P-18 and P-34 xenografts. Means and standard deviations (error bar) were determined from six replicates $(n=6)$. Error bars represent mean \pm SD for triplicate experiments. NS, no significance.

\section{Additional file 2.}

Additional file 3.

Additional file 4.

Additional file $\mathbf{5}$.

\section{Abbreviations}

m6A: N6-methyladenosine; NEAT1: Nuclear-enriched abundant transcript 1; ncRNA: noncoding RNA; CDK : Cyclin-dependent kinase; CLIP: Cross-linking immunoprecipitation; PCR: Polymerase chain reaction; qPCR: Quantitative real-time PCR; RBR: RNA binding residues; RIP: RNA immunoprecipitation; 3' UTR: 3'-untranslated region

\section{Acknowledgements}

We thank the patients and their families for their altruism in participating in research studies. We also thank Dr. Wei Xiong for reagents, plasmids, and suggestions about AR and NEAT1-1. This work was supported in part by grants from the National Natural Science Foundation of China (81972654 to S.W.), Tianjin International Student Science and Technology Activities (20160014 to S.W.), Individualized funding (to Y.Z.).

\section{Authors' contributions}

SW and YW performed the experiments. SW, YW and YZ prepared all the figures and wrote the manuscript. SW, CZ and WX collected the RCC samples. $Y Z$ and $Y N$ supervised the project. SW and $Y N$ provided fund for the whole project. All authors read and approved the final manuscript.

\section{Availability of data and materials}

All data that support the findings of this study are available from the corresponding authors upon reasonable request.

\section{Competing interest}

'The authors declare no conflict of interest.

\section{Author details}

${ }^{1}$ Department of Urology, The Second Hospital of Tianjin Medical University, Tianjin Medical University, Tianjin 300211, China. ${ }^{2}$ Department of Gynecology and Obstetrics, Tianjin First Central Hospital, Tianjin 300192, China. ${ }^{3}$ Department of Urology, Central South University, Changsha 410011, China. ${ }^{4}$ Department of Biochemistry and Molecular Biology, Mayo Clinic, Rochester, MN 55905, USA

Received: 10 June 2020 Accepted: 6 December 2020

Published online: 12 December 2020

\section{References}

1. Siegel RL, Miller KD, Jemal A. Cancer statistics, 2016. CA Cancer J Clin. 2016; 66:7-30.

2. van der Toom EE, Verdone JE, Pienta KJ. Disseminated tumor cells and dormancy in prostate cancer metastasis. Curr Opin Biotechnol. 2016;40:9-15. 
3. van der Toom EE, Axelrod HD, de la Rosette JJ, de Reijke TM, Pienta KJ, Valkenburg KC. Prostate-specific markers to identify rare prostate cancer cells in liquid biopsies. Nat Rev Urol. 2019;16:7-22.

4. Yin JJ, Pollock CB, Kelly K. Mechanisms of cancer metastasis to the bone. Cell Res. 2005;15:57-62.

5. Smith MR, Cook R, Lee KA, Nelson JB. Disease and host characteristics as predictors of time to first bone metastasis and death in men with progressive castration-resistant nonmetastatic prostate cancer. Cancer. 2011 117:2077-85.

6. Norgaard M, Jensen AO, Jacobsen JB, Cetin K, Fryzek JP, Sorensen HT Skeletal related events, bone metastasis and survival of prostate cancer: a population based cohort study in Denmark (1999 to 2007). J Urol. 2010;184: 162-7.

7. Dominissini D, Moshitch-Moshkovitz S, Schwartz S, Salmon-Divon M, Ungar L, Osenberg S, Cesarkas K, Jacob-Hirsch J, Amariglio N, Kupiec M, et al. Topology of the human and mouse m6A RNA methylomes revealed by m6A-seq. Nature. 2012;485:201-6.

8. Meyer KD, Saletore Y, Zumbo P, Elemento O, Mason CE, Jaffrey SR. Comprehensive analysis of mRNA methylation reveals enrichment in 3' UTRs and near stop codons. Cell. 2012;149:1635-46.

9. Fu Y, Dominissini D, Rechavi $G$, He C. Gene expression regulation mediated through reversible m (6) a RNA methylation. Nat Rev Genet. 2014;15:293306.

10. Meyer KD, Jaffrey SR. The dynamic epitranscriptome: N6-methyladenosine and gene expression control. Nat Rev Mol Cell Biol. 2014;15:313-26.

11. Wang X, Lu Z, Gomez A, Hon GC, Yue Y, Han D, Fu Y, Parisien M, Dai Q, Jia $G$, et al. N6-methyladenosine-dependent regulation of messenger RNA stability. Nature. 2014;505:117-20.

12. Wang X, Zhao BS, Roundtree IA, Lu Z, Han D, Ma H, Weng X, Chen K, Shi H, He C. N (6)-methyladenosine modulates messenger RNA translation efficiency. Cell. 2015;161:1388-99.

13. Zhao $X$, Yang $Y$, Sun BF, Shi $Y$, Yang $X$, Xiao W, Hao YJ, Ping XL, Chen YS, Wang WJ, et al. FTO-dependent demethylation of N6-methyladenosine regulates mRNA splicing and is required for adipogenesis. Cell Res. 2014;24: 1403-19.

14. Wang Y, Li Y, Toth Jl, Petroski MD, Zhang Z, Zhao JC. N6-methyladenosine modification destabilizes developmental regulators in embryonic stem cells. Nat Cell Biol. 2014;16:191-8.

15. Dong P, Xiong Y, Yue J, Hanley SJB, Kobayashi N, Todo Y, Watari H. Long non-coding RNA NEAT1: a novel target for diagnosis and therapy in human tumors. Front Genet. 2018;9:471.

16. Lanzos A, Carlevaro-Fita J, Mularoni L, Reverter F, Palumbo E, Guigo R, Johnson R. Discovery of Cancer driver Long noncoding RNAs across 1112 tumour genomes: new candidates and distinguishing features. Sci Rep. 2017;7:41544.

17. Zhao D, Zhang Y, Wang N, Yu N. NEAT1 negatively regulates miR-218 expression and promotes breast cancer progression. Cancer Biomark. 2017 20:247-54.

18. Li W, Zhang Z, Liu X, Cheng X, Zhang Y, Han X, Zhang Y, Liu S, Yang J, Xu $B$, et al. The FOXN3-NEAT1-SIN3A repressor complex promotes progression of hormonally responsive breast cancer. J Clin Invest. 2017;127:3421-40.

19. Fujimoto A, Furuta M, Totoki Y, Tsunoda T, Kato M, Shiraishi Y, Tanaka H, Taniguchi $\mathrm{H}$, Kawakami $Y$, Ueno M, et al. Whole-genome mutational landscape and characterization of noncoding and structural mutations in liver cancer. Nat Genet. 2016:48:500-9.

20. Wang P, Wu T, Zhou H, Jin Q, He G, Yu H, Xuan L, Wang X, Tian L, Sun Y, et al. Long noncoding RNA NEAT1 promotes laryngeal squamous cell cancer through regulating miR-107/CDK6 pathway. J Exp Clin Cancer Res. 2016:35:22.

21. Sun C, Li S, Zhang F, Xi Y, Wang L, Bi Y, Li D. Long non-coding RNA NEAT1 promotes non-small cell lung cancer progression through regulation of miR-377-3p-E2F3 pathway. Oncotarget. 2016;7:51784-814

22. Zhen $L$, Yun-Hui L, Hong-Yu D, Jun M, Yi-Long Y. Long noncoding RNA NEAT1 promotes glioma pathogenesis by regulating miR-449b-5p/c-met axis. Tumour Biol. 2016;37:673-83

23. Chakravarty D, Sboner A, Nair SS, Giannopoulou E, Li R, Hennig S, Mosquera JM, Pauwels J, Park K, Kossai M, et al. The oestrogen receptor alpharegulated IncRNA NEAT1 is a critical modulator of prostate cancer. Nat Commun. 2014;5:5383.

24. Adriaens C, Standaert L, Barra J, Latil M, Verfaillie A, Kalev P, Boeckx B, Wijnhoven PW, Radaelli E, Vermi W, et al. p53 induces formation of NEAT1
IncRNA-containing paraspeckles that modulate replication stress response and chemosensitivity. Nat Med. 2016;22:861-8.

25. Clemson CM, Hutchinson JN, Sara SA, Ensminger AW, Fox AH, Chess A, Lawrence JB. An architectural role for a nuclear noncoding RNA: NEAT1 RNA is essential for the structure of paraspeckles. Mol Cell. 2009;33:717-26.

26. Hirose T, Virnicchi G, Tanigawa A, Naganuma T, Li R, Kimura H, Yokoi T, Nakagawa S, Benard M, Fox AH, Pierron G. NEAT1 long noncoding RNA regulates transcription via protein sequestration within subnuclear bodies. Mol Biol Cell. 2014;25:169-83.

27. West JA, Davis CP, Sunwoo H, Simon MD, Sadreyev RI, Wang PI, Tolstorukov MY, Kingston RE. The long noncoding RNAs NEAT1 and MALAT1 bind active chromatin sites. Mol Cell. 2014;55:791-802.

28. Bond CS, Fox AH. Paraspeckles: nuclear bodies built on long noncoding RNA. J Cell Biol. 2009;186:637-44.

29. Idogawa M, Ohashi T, Sasaki Y, Nakase H, Tokino T. Long non-coding RNA NEAT1 is a transcriptional target of p53 and modulates p53-induced transactivation and tumor-suppressor function. Int J Cancer. 2017;140:278591.

30. Mello SS, Sinow C, Raj N, Mazur PK, Bieging-Rolett K, Broz DK, Imam JFC, Vogel H, Wood LD, Sage J, et al. Neat1 is a p53-inducible lincRNA essential for transformation suppression. Genes Dev. 2017;31:1095-108.

31. Xiong W, Huang C, Deng H, Jian C, Zen C, Ye K, Zhong Z, Zhao X, Zhu L. Oncogenic non-coding RNA NEAT1 promotes the prostate cancer cell growth through the SRC3/IGF1R/AKT pathway. Int J Biochem Cell Biol. 2018; 94:125-32.

32. Zhao Y, Peng J, Yang J, Zhang E, Huang L, Yang H, Kakadiaris E, Li J, Yan B, Shang Z, et al. Enhancing prostate-Cancer-specific MRI by genetic amplified nanoparticle tumor homing. Adv Mater. 2019;31:e1900928.

33. Shang Z, Yu J, Sun L, Tian J, Zhu S, Zhang B, Dong Q, Jiang N, FloresMorales A, Chang C, Niu Y. LncRNA PCAT1 activates AKT and NF-kappaB signaling in castration-resistant prostate cancer by regulating the PHLPP/ FKBP51/IKKalpha complex. Nucleic Acids Res. 2019;47:4211-25.

34. Zhao Y, Wang L, Ren S, Wang L, Blackburn PR, McNulty MS, Gao X, Qiao M, Vessella RL, Kohli M, et al. Activation of P-TEFb by androgen receptorregulated enhancer RNAs in castration-resistant prostate Cancer. Cell Rep. 2016;15:599-610.

35. Vaubel RA, Tian S, Remonde D, Schroeder MA, Mladek AC, Kitange GJ, Caron A, Kollmeyer TM, Grove R, Peng S, et al. Genomic and phenotypic characterization of a broad panel of patient-derived Xenografts reflects the diversity of Glioblastoma. Clin Cancer Res. 2020;26:1094-104.

36. Seth PP, Siwkowski A, Allerson CR, Vasquez G, Lee S, Prakash TP, Kinberger G, Migawa MT, Gaus H, Bhat B, Swayze EE. Design, synthesis and evaluation of constrained methoxyethyl (CMOE) and constrained ethyl (cEt) nucleoside analogs. Nucleic Acids Symp Ser (Oxf). 2008;52:553-4.

37. Zhao Y, Ding L, Wang D, Ye Z, He Y, Ma L, Zhu R, Pan Y, Wu Q, Pang K, et al. EZH2 cooperates with gain-of-function p53 mutants to promote cancer growth and metastasis. EMBO J. 2019;38:e99599.

38. Linder B, Grozhik AV, Olarerin-George AO, Meydan C, Mason CE, Jaffrey SR Single-nucleotide-resolution mapping of $\mathrm{m} 6 \mathrm{~A}$ and $\mathrm{m} 6 \mathrm{Am}$ throughout the transcriptome. Nat Methods. 2015;12:767-72.

39. Chen K, Luo GZ, He C. High-resolution mapping of N (6)-Methyladenosine in Transcriptome and genome using a photo-crosslinking-assisted strategy. Methods Enzymol. 2015;560:161-85.

40. Cui Q, Shi H, Ye P, Li L, Qu Q, Sun G, Sun G, Lu Z, Huang Y, Yang CG, et al. $m$ (6) a RNA methylation regulates the self-renewal and tumorigenesis of Glioblastoma stem cells. Cell Rep. 2017;18:2622-34.

41. Zhang Y, Pitchiaya S, Cieslik M, Niknafs YS, Tien JC, Hosono Y, lyer MK, Yazdani S, Subramaniam S, Shukla SK, et al. Analysis of the androgen receptor-regulated IncRNA landscape identifies a role for ARLNC1 in prostate cancer progression. Nat Genet. 2018;50:814-24.

42. Wu B, Su S, Patil DP, Liu H, Gan J, Jaffrey SR, Ma J. Molecular basis for the specific and multivariant recognitions of RNA substrates by human hnRNP A2/B1. Nat Commun. 2018;9:420.

43. Lu Z, Zhang QC, Lee B, Flynn RA, Smith MA, Robinson JT, Davidovich C, Gooding AR, Goodrich KJ, Mattick JS, et al. RNA duplex map in living cells reveals higher-order Transcriptome structure. Cell. 2016; 165:1267-79.

44. Lin Y, Schmidt BF, Bruchez MP, McManus CJ. Structural analyses of NEAT1 IncRNAs suggest long-range RNA interactions that may contribute to paraspeckle architecture. Nucleic Acids Res. 2018;46:3742-52. 
45. Clark MB, Johnston RL, Inostroza-Ponta M, Fox AH, Fortini E, Moscato P, Dinger ME, Mattick JS. Genome-wide analysis of long noncoding RNA stability. Genome Res. 2012;22:885-98.

46. Friedel CC, Dolken L, Ruzsics Z, Koszinowski UH, Zimmer R. Conserved principles of mammalian transcriptional regulation revealed by RNA half-life. Nucleic Acids Res. 2009;37:e115.

47. Adriaens C, Rambow F, Bervoets G, Silla T, Mito M, Chiba T, Asahara H, Hirose T, Nakagawa S, Jensen TH, Marine JC. The long noncoding RNA NEAT1_1 is seemingly dispensable for normal tissue homeostasis and cancer cell growth. RNA. 2019;25:1681-95.

48. Kiledjian M, Dreyfuss G. Primary structure and binding activity of the hnRNP U protein: binding RNA through RGG box. EMBO J. 1992;11:2655-64.

49. Maris C, Dominguez C, Allain FH. The RNA recognition motif, a plastic RNAbinding platform to regulate post-transcriptional gene expression. FEBS J. 2005;272:2118-31.

50. Tan R, Frankel AD. Structural variety of arginine-rich RNA-binding peptides. Proc Natl Acad Sci U S A. 1995;92:5282-6.

51. Donner AJ, Ebmeier CC, Taatjes DJ, Espinosa JM. CDK8 is a positive regulator of transcriptional elongation within the serum response network. Nat Struct Mol Biol. 2010;17:194-201.

52. Zaborowska J, Egloff S, Murphy S. The pol II CTD: new twists in the tail. Nat Struct Mol Biol. 2016:23:771-7.

53. Bragelmann J, Klumper N, Offermann A, von Massenhausen A, Bohm D, Deng M, Queisser A, Sanders C, Syring I, Merseburger AS, et al. Pan-Cancer analysis of the mediator complex Transcriptome identifies CDK19 and CDK8 as therapeutic targets in advanced prostate Cancer. Clin Cancer Res. 2017:23:1829-40.

54. Lellahi SM, Rosenlund IA, Hedberg A, Kiaer LT, Mikkola I, Knutsen E, Perander M. The long noncoding RNA NEAT1 and nuclear paraspeckles are upregulated by the transcription factor HSF1 in the heat shock response. J Biol Chem. 2018;293:18965-76.

55. Zhang P, Cao L, Zhou R, Yang X, Wu M. The IncRNA Neat1 promotes activation of inflammasomes in macrophages. Nat Commun. 2019;10:1495.

56. Yin QF, Hu SB, Xu YF, Yang L, Carmichael GG, Chen LL. SnoVectors for nuclear expression of RNA. Nucleic Acids Res. 2015;43:e5.

57. van den Hoogen C, van der Horst G, Cheung H, Buijs JT, Lippitt JM, GuzmanRamirez N, Hamdy FC, Eaton CL, Thalmann GN, Cecchini MG, et al. High aldehyde dehydrogenase activity identifies tumor-initiating and metastasis-initiating cells in human prostate cancer. Cancer Res. 2010;70:5163-73.

58. Baniwal SK, Khalid O, Gabet Y, Shah RR, Purcell DJ, Mav D, Kohn-Gabet AE, Shi Y, Coetzee GA, Frenkel B. Runx2 transcriptome of prostate cancer cells: insights into invasiveness and bone metastasis. Mol Cancer. 2010;9:258.

59. Ozdemir BC, Hensel J, Secondini C, Wetterwald A, Schwaninger R, Fleischmann A, Raffelsberger W, Poch O, Delorenzi M, Temanni R, et al. The molecular signature of the stroma response in prostate cancer-induced osteoblastic bone metastasis highlights expansion of hematopoietic and prostate epithelial stem cell niches. PLoS One. 2014;9:e114530.

60. Akech J, Wixted JJ, Bedard K, van der Deen M, Hussain S, Guise TA, van Wijnen AJ, Stein JL, Languino LR, Altieri DC, et al. Runx2 association with progression of prostate cancer in patients: mechanisms mediating bone osteolysis and osteoblastic metastatic lesions. Oncogene. 2010;29:811-21.

61. Zhao JC, Yu J, Runkle C, Wu L, Hu M, Wu D, Liu JS, Wang Q, Qin ZS, Yu J. Cooperation between Polycomb and androgen receptor during oncogenic transformation. Genome Res. 2012;22:322-31.

62. Cohen-Solal KA, Boregowda RK, Lasfar A. RUNX2 and the PI3K/AKT axis reciprocal activation as a driving force for tumor progression. Mol Cancer. 2015;14:137.

63. Liu N, Dai Q, Zheng G, He C, Parisien M, Pan T. N (6)-methyladenosinedependent RNA structural switches regulate RNA-protein interactions. Nature. 2015;518:560-4.

64. Patil DP, Chen CK, Pickering BF, Chow A, Jackson C, Guttman M, Jaffrey SR. m (6) a RNA methylation promotes XIST-mediated transcriptional repression. Nature. 2016;537:369-73.

65. Loyer P, Trembley JH, Grenet JA, Busson A, Corlu A, Zhao W, Kocak M, Kidd VJ, Lahti JM. Characterization of cyclin L1 and L2 interactions with CDK11 and splicing factors: influence of cyclin $L$ isoforms on splice site selection. J Biol Chem. 2008;283:7721-32.

\section{Publisher's Note}

Springer Nature remains neutral with regard to jurisdictional claims in published maps and institutional affiliations.

\section{Ready to submit your research? Choose BMC and benefit from:}

- fast, convenient online submission

- thorough peer review by experienced researchers in your field

- rapid publication on acceptance

- support for research data, including large and complex data types

- gold Open Access which fosters wider collaboration and increased citations

- maximum visibility for your research: over $100 \mathrm{M}$ website views per year

At BMC, research is always in progress.

Learn more biomedcentral.com/submissions 